March 2001

UCOFIS $1 / 01$

US-FT/2-01

LPT Orsay 01-15

\title{
Monte Carlo model for nuclear collisions from SPS to LHC energies
}

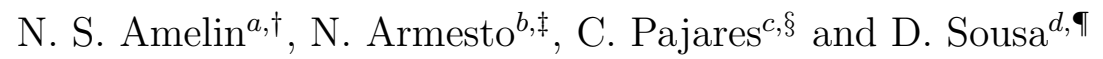 \\ a Department of Physics, University of Jyväskylä, \\ P.O.Box 35, FIN-40351 Jyväskylä, Finland \\ ${ }^{b}$ Departamento de Física, Módulo C2, Planta baja, Campus de Rabanales, \\ Universidad de Córdoba, E-14071 Córdoba, Spain \\ ${ }^{c}$ Departamento de Física de Partículas, Universidade de Santiago de Compostela, \\ E-15706 Santiago de Compostela, Spain \\ d Laboratoire de Physique Théorique, Université de Paris XI, \\ Bâtiment 210, F-91405 Orsay Cedex, France \\ † E-mail: nikolai.ameline@phys.jyu.fi \\ $\ddagger$ E-mail: fa1arpen@uco.es \\ $\S$ E-mail: pajares@fpaxp1.usc.es \\ ฯ E-mail: Dolores.Sousa@th.u-psud.fr
}

\begin{abstract}
A Monte Carlo model to simulate nuclear collisions in the energy range going from SPS to LHC, is presented. The model includes in its initial stage both soft and semihard components, which lead to the formation of color strings. Collectivity is taken into account considering the possibility of strings in color representations higher than triplet or antitriplet, by means of string fusion. String breaking leads to the production of secondaries. At this point, the model can be used as initial condition for further evolution by a transport model. In order to tune the parameters and see the results in nucleus-nucleus collisions, a naif model for rescattering of secondaries is introduced. Results of the model are compared with experimental data, and predictions for RHIC and LHC are shown.
\end{abstract}




\section{Introduction}

With the announcement of the discovery of Quark Gluon Plasma (QGP) at the Super Proton Synchrotron (SPS) at CERN [1], the experimental heavy ion program moves now to the higher energies of the Relativistic Heavy Ion Collider (RHIC) at Brookhaven and the Large Hadron Collider (LHC) at CERN. Whether this claim can be considered conclusive or not (see e.g. [2]), the most compelling experimental findings at the SPS [3, 4, 5, 6, 7, 8, 9] are interpreted as positive signatures of QGP only when conventional, non-QGP models fail to reproduce them. Therefore, even in the case that QGP has already been obtained, it is most important that conventional models employed at the SPS become generalized for RHIC and LHC: They can be used to describe collisions between less massive nuclei or more peripheral events than those in which QGP is expected, and to establish the background to events with QGP production.

On the other hand, the situation with conventional models is not clear at all. The description of a high energy collision between heavy ions is a complex task which involves different physical aspects. Predictions from different models for RHIC and LHC are far from being compatible, see the reviews [10 and [11]. For example, the values for central rapidity densities of charged particles coming from different models lie in the ranges $600 \div 1500$ for central AuAu collisions at RHIC and $2000 \div 8000$ for central $\mathrm{PbPb}$ collisions at LHC.

In this paper a non-QGP model for collisions between nucleons or nuclei in the energy range going from SPS energies $(\sim 20 \mathrm{GeV}$ per nucleon in the center of mass $)$ to LHC energies (5.5 TeV per nucleon in the center of mass) is presented (different steps in this direction can be found in [12, 13]). The model is based on the ideas of Dual Parton Model (DPM) [14 or Quark-Gluon String Model (QGSM) [15], considering both soft and semihard components on a partonic level. These elementary partonic collisions lead to the formation of color strings. Collectivity is taken into account considering the possibility of strings in color representations higher than triplet or antitriplet, by means of string fusion, as done in [12, 16] (see related approaches in [17, 18]). String breaking leads to the production of secondaries. In this form, the model can be used as initial condition for subsequent evolution using a transport model, as those of [16, 17]. Nevertheless, in order to tune the parameters of the model and apply it to nucleusnucleus collisions, rescattering between secondaries is considered on the basis of $2 \longrightarrow 2$ collisions, using a very simple model which allows us just to estimate the effects of such 
process. The results of the code turn out to agree reasonably with existing experimental data on total multiplicities, and longitudinal and transverse momentum distributions, and semiquantitatively with strangeness production and stopping power.

The paper is organized as follows: In Section 2 string formation will be discussed, both for soft and semihard components, whose separation will be established. Also in this Section collectivity, considered as string interaction or fusion, will be presented. Hadronization of the produced strings will be formulated in Section 3 . In Section 4 our simple approach to rescattering between secondaries will be presented. A comparison with experimental data will be done in Section 5, and predictions for RHIC and LHC shown in Section 6, together with some discussion on the first RHIC data [19, 20]. In the last Section we will summarize our conclusions and briefly compare with other approaches.

\section{Initial stage}

\subsection{Elementary partonic collisions}

To compute the number of elementary partonic collisions we have to generate the partonic wave functions of the colliding hadrons. The steps to generate this wave function for the projectile $A$ and target $B$ are the following: First, the impact parameter $b$ of the collision is generated uniformly between 0 and $R_{A}+R_{B}$ (in the case of nucleons, the total cross section determines the corresponding radius).

Second, the nuclear wave function is computed. Nucleon positions inside the nucleus are distributed in transverse space according to a Woods-Saxon distribution for $A>11$,

$$
\rho(r) \propto \frac{1}{1+\exp \left[\left(r-r_{n}\right) / a\right]},
$$

with $r_{n}=1.07 A^{1 / 3} \mathrm{fm}$ and $a=0.545 \mathrm{fm}$, and according to a Gaussian distribution for $A \leq 11$, with parameters chosen for each nucleus [21]. Then, Fermi motion is given to the nucleons in the nuclei uniformly in the range $0<p<p_{F}$, with the maximum Fermi momentum given in the local Thomas-Fermi approximation [22 by

$$
p_{F}=h[3 \pi \rho(r)]^{1 / 3},
$$

with $h=0.197 \mathrm{fm} \mathrm{GeV} / \mathrm{c}$. 
Now partons are generated inside each nucleon. Its number is given by a Poisson distribution [23],

$$
W_{N} \propto \exp (-g(s) C / 2) \frac{[C g(s) / 2]^{N}}{C N ! / 2}, g(s)=g_{0} s^{\Delta / 2}, \quad g_{0}=\frac{8 \pi \gamma_{P}}{\sigma_{P}},
$$

with $\Delta=0.139$ the pomeron intercept minus $1, C=3.0$ the quasieikonal parameter which takes into account low mass nucleon dissociation, $\gamma_{P}=1.77 \mathrm{GeV}^{2}$ the pomeronnucleon vertex, $\sigma_{P}=3.3 \mathrm{mb}$ the parton-parton cross section and $\sqrt{s}$ the center of mass energy for each nucleon-nucleon collision.

Parton positions in transverse space (inside a nucleon) are given by a Gaussian according to Regge theory,

$$
F(b)=\frac{1}{4 \pi \lambda} \exp \left(-\frac{b^{2}}{4 \lambda}\right), \quad \lambda=R_{0}^{2}+\alpha^{\prime} \ln s,
$$

with $R_{0}^{2}=3.18 \mathrm{GeV}^{2}$ and $\alpha^{\prime}=0.21 \mathrm{GeV}^{2}$ the pomeron slope.

Now, one parton from the projectile and one from the target produce an inelastic collision if both are within an area in impact parameter equal to $\sigma_{P}=2 \pi r_{P}^{2}, r_{P}=0.23$ fm. In this way, events with no inelastic collisions are elastic, while those with at least one inelastic collision are inelastic. Taking the total cross section given by the quasieikonal model [24]

$$
\sigma_{\text {tot }}=\sigma_{P} f(z / 2), \quad z=\frac{C \gamma_{P} s^{\Delta}}{\lambda}, \quad f(z)=\sum_{k=1}^{\infty} \frac{(-z)^{k-1}}{k \cdot k !},
$$

all cross sections can be computed, see next Subsection (all formulae reduce to the usual eikonal ones with $C=2$ ).

\subsection{Semihard component}

The inclusion of semihard components, in the form of a two-component model, is needed to reproduce the $p_{T}$ spectra in hadronic collisions, see Section 5 . In the model this is performed considering that an inelastic collision is hard with probability

$$
W_{h}=\frac{C_{h}\left(s-s_{0}\right)^{\Delta_{h}}}{C_{h}\left(s-s_{0}\right)^{\Delta_{h}}+s^{\Delta}},
$$

with $\Delta_{h}=0.50, \sqrt{s_{0}}=25 \mathrm{GeV}$ and $C_{h}=0.0035$. A hard collision proceeds through the packages PYTHIA 5.5 + ARIADNE 4.02 + JETSET 7.3 [25, 26]. Only gluongluon collisions are included in PYTHIA, and the key parameter here is the cut-off in transverse momentum

$$
p_{\text {Tmin }}=3.03+0.11 \ln \left(s / s_{0}\right) \mathrm{GeV} / \mathrm{c} \text {. }
$$


The minimum energy for an elementary collision to be accepted by PYTHIA is 20 $\mathrm{GeV}$, and for the global collision the minimum center of mass energy per nucleon is $\sqrt{s_{0}}=25 \mathrm{GeV}$. An event is considered hard if at least one of its inelastic elementary collisions successfully proceeds through PYTHIA.

While the concrete choice of the parameters in $p_{T \text { min }}$ comes from a fit to experimental data, let us make some comments on its functional form. In our case, an increase of $p_{\text {Tmin }}$ with increasing energy makes possible a smooth transition from the soft to the semihard part of the $p_{T}$ spectrum. Usually $p_{T \text { min }}$ is taken as either constant or increasing as a polynomial of a logarithm of $s$ [27, 28]. It may be argued that the $p_{\text {Tmin }}$ value which indicates the transition from nonperturbative to perturbative QCD (pQCD), is related with the proposed saturation scale $Q_{s}^{2}$ [29, 30]: below this $Q_{s}^{2}$, the number of partons in the hadron wave function cannot grow, as new partons fuse with the existing ones and cannot be resolved individually. Nevertheless, apart from conceptual differences, the dependences of $p_{T \min }$ and $Q_{s}^{2}$ are not the same: while the first depends only on energy, the second one also depends on the size of the colliding objects $\left(Q_{s}^{2} \propto A^{\alpha}, \alpha=1 / 3 \div 2 / 3\right)$.

Results of the model for the total, inelastic (production) and hard cross sections in $\mathrm{pp}$ and $\overline{\mathrm{p}} \mathrm{p}$ collisions at different energies are shown in Fig. 11 and compared with experimental data for the total cross section [31]. It can be observed that both the total and the production cross section are too small at low energies, while they get reasonable values at higher energies. The reasons for the existing discrepancies are three: In first place, diffraction is not properly included in the model, so it is difficult to distinguish between production and inelastic cross sections. In second place, no reggeon contribution (decreasing with energy) has been included. In third place, at the level of the cross sections no distinction is made between nucleons and antinucleons as projectiles and targets. These two last reasons should improve the agreement with data at energies of SPS and Intersecting Storage Rings (ISR). Also in this Figure it is

shown the value of $p_{\text {Tmin }}$ and the mean number of total and hard inelastic collisions per event.

\subsection{String formation and fusion}

Each soft parton-parton collision gives rise to two strings [14, 15], stretched either between valence quarks and diquarks (for the first collision suffered by a nucleon) 
or sea quarks and antiquarks (for the subsequent ones). For the latter, their flavors follow the ratio $u: d: s=1: 1: 0.26$. Hard collisions proceed through PYTHIA as $g g \longrightarrow g g$. For the string ends and hard gluons, the longitudinal momentum fractions are distributed as

$$
L\left(x_{1}, x_{2}, \ldots, x_{n}\right)=f_{q q}\left(x_{1}\right) f_{q}\left(x_{2}\right) \cdots f_{q}\left(x_{n}\right), \quad \sum_{k=1}^{n} x_{k}=1 .
$$

For soft strings ends, the individual momentum distributions are those of the QGSM |15],

$$
f_{q q}(x)=x^{3 / 2}, \quad f_{q(\bar{q})}(x)=\frac{1}{\sqrt{x}},
$$

with a lower cut-off $x_{\text {min }}=0.3 \mathrm{GeV} / \sqrt{s_{N N}}$ to ensure that the strings have mass enough to be projected onto hadrons, $\sqrt{s_{N N}}$ being the center of mass energy per nucleon.

For partons involved in hard collisions, the longitudinal momentum fractions are taken by PYTHIA from PDFLIB [32], with the possibility of considering the difference of parton distributions inside nuclei given by the parametrization EKS98 [33] or by a parametrization as $F_{2 A}$ [34. After generating the final gluons, each of them splits into a $(q \bar{q})$ pair and strings are stretched between them, according with the standard procedure in PYTHIA [25].

The transverse momentum of both partons at the string ends and hard partons, coming from a nucleon which has been wounded $m$ times, is given by a Gaussian:

$$
T\left(p_{\perp}\right)=\frac{1}{\pi \delta^{2}} \exp \left(-p_{\perp}^{2} / \delta^{2}\right), \quad \delta=0.5 \sqrt{m} \mathrm{GeV} / \mathrm{c} ;
$$

in this way, $p_{T}$-broadening is taken into account [35].

The number of strings exchanged in one collision is quite low for nucleon-nucleon collisions, but this number increases with energy, size of projectile and target and centrality of the collisions. Strings can be viewed as objects with a certain area, given by the uncertainty relation as $\propto 1 /\left\langle p_{T}^{2}\right\rangle$, in the transverse plane of the collision. When the number of strings is high enough, they begin to overlap and the usual hypothesis in QGSM or DPM of the strings being independent sources of secondary particles is expected to break down. A possible way of considering this is to compute the density of strings in the transverse plane and use two-dimensional percolation as an indicator of the onset of collectivity [36, 37]. Percolation takes place when domains of overlapping strings acquire a size of the order of the total available size for the collision.

While percolation is a second order phase transition, the option we use in this model, fusion of strings, does not lead to any phase transition [38. In the model, 
ordinary strings (i.e. in a triplet representation of $\mathrm{SU}(3)$ ) fuse $\mathrm{\text {in }}$ pairs when their parent partons (those which determined the inelastic collision the strings come from) are within a certain area $\sigma_{f u s}=2 \pi r_{f u s}^{2}$ in impact parameter space. In the code we consider only fusion of two strings but there is a probability of fusion of more than two. An effective way of taking this into account is to increase the cross section for the fusion of two strings, for which we will take $\sigma_{P}<\sigma_{\text {fus }}=7.5 \mathrm{mb}\left(r_{\text {fus }}=0.35 \mathrm{fm}\right)$. This value is crucial to reproduce the strangeness enhancement in central SS and SAg collisions at SPS [39]. The fusion can take place only when the rapidity intervals of the strings overlap. It is formally described by allowing partons to interact several times, the number of interactions being the same both for projectile and target.

The quantum numbers of the fused strings are determined by the interacting partons and their energy-momentum is the sum of the energy-momenta of their ancestor strings. The color charge of the resulting string ends is obtained according to the $\mathrm{SU}(3)$ composition laws:

$$
\{3\} \otimes\{3\}=\{6\} \oplus\{\overline{3}\}, \quad\{3\} \otimes\{\overline{3}\}=\{1\} \oplus\{8\} .
$$

Thus, two triplet strings fuse into either a sextet or an antitriplet string with probabilities $2 / 3$ and $1 / 3$ respectively, and one triplet and one antitriplet string fuse into either a singlet or an octet string with probabilities $1 / 9$ and $8 / 9$ respectively.

Two comments are in order: On the one hand and as written above, the fusion of strings means nothing related to a phase transition. On the contrary, percolation of strings [36] is a non-thermal second order phase transition. In this case, the key parameter is $\eta=\pi r^{2} N /\left(\pi R_{A}^{2}\right)$, which is the density of strings $N /\left(\pi R_{A}^{2}\right)$ (number of strings $N$ produced in the overlapping area of the collision, $\pi R_{A}^{2}$ for central collisions) times the transverse size of one string $\pi r^{2}$. The critical point for percolation is $\eta_{c} \simeq$ $1.12 \div 1.5$ depending on the profile function of the colliding nuclei [37]. With $r \simeq$ $0.2 \div 0.25 \mathrm{fm}$, this critical value means $6 \div 12$ strings $/ \mathrm{fm}^{2}$. The value of 9 is reached in central $\mathrm{PbPb}$ collisions at $\mathrm{SPS}$, in central $\mathrm{AgAg}$ collisions at RHIC and in central SS collisions at LHC. We expect for $\eta$ around or greater than $\eta_{c}$, that the approximation of fusion of just two strings fails. On the other hand, only fusion of soft strings is considered. Hard strings are not fused, their area being proportional to $1 / p_{T}^{2}$. Some effect of the fusion of such strings could appear at LHC energies where, for instance, in central $\mathrm{PbPb}$ collisions they amount for $32 \%$ of the binary nucleon-nucleon collisions.

\footnotetext{
${ }^{1}$ A similar mechanism exists in RQMD [16], called color ropes.
} 


\section{Hadronization}

Now we consider the breaking of a soft string with color charges $Q$ and $\bar{Q}$ in its ends (corresponding to a representation $\{N\}$ of $\mathrm{SU}(3)$ ). In our model, it is due to the production of two (anti)quark complexes with the same color charges $Q$ and $\bar{Q}$ as those at the ends of the string $[12$. The probability rate is given by the Schwinger formula 40

$$
W \propto K_{\{N\}}^{2} \exp \left(-\pi M_{T}^{2} / K_{\{N\}}\right),
$$

where $K_{\{N\}}$ is the string tension for the $\{N\}$ representation, proportional to the corresponding quadratic Casimir operator $C_{\{N\}}^{2}$ (as found both in lattice QCD and in the Stochastic Vacuum Model [41, 42]), i.e.

$$
K_{\{N\}}=K_{\{3\}} \frac{C_{\{N\}}^{2}}{C_{\{3\}}^{2}}, \quad C_{\{3\}}^{2}=4 / 3, \quad C_{\{6\}}^{2}=10 / 3, \quad C_{\{8\}}^{2}=3 .
$$

For the longitudinal breaking of the string, an invariant area law [43] is employed,

$$
P \propto \exp (-b A), \quad K_{\{N\}} \propto b C_{\{N\}}^{2}, \quad A=p_{+} p_{-}
$$

being the area in light-cone momentum space determined by the breaking point in the center of mass frame of the string. This law gives results quite similar to those of the Lund model 44] implemented in JETSET 25].

We proceed as follows: Eq. (12) is used to decide the flavors of the quark and antiquark complexes created. We take $K_{\{3\}}=0.18 \mathrm{GeV}^{2}$ and $m_{u}=m_{d}=0.23 \mathrm{GeV} / \mathrm{c}^{2}$, $m_{s}=0.35 \mathrm{GeV} / \mathrm{c}^{2}$, and the masses of a complex $\left(q_{1} \ldots q_{l}\right)$ is given by $M\left(q_{1} \ldots q_{l}\right)=$ $\sum_{i=1}^{l} m_{q i}$. Then $p_{T}$ is given to one of the created complexes and $-p_{T}$ to the other one, according to a Gaussian law

$$
f\left(p_{T}^{2}\right) \propto \exp \left(-\alpha_{\{N\}} p_{T}^{2}\right)
$$

with $\alpha_{\{3\}}=\alpha_{\{\overline{3}\}}=4 \mathrm{GeV}^{-2}$ and

$$
\alpha_{\{N\}}=2 \alpha_{\{3\}} \frac{C_{\{3\}}^{2}}{C_{\{N\}}^{2}}, \quad\{N\} \neq\{3\},\{\overline{3}\} .
$$

\footnotetext{
${ }^{2}$ This possibility is the dominant one for strings formed by fusion of two triplet strings 12. For higher color representations, production of quark complexes with color charges $Q^{\prime}<Q$ begins to dominate. This option is taken in RQMD [16]; nevertheless, the close similarities in the consequences of string fusion in both approaches, strongly suggest that the difference in the breaking mechanism can be compensated by a different choice of the fragmentation parameters.
} 
Finally a breaking point is sampled according to Eq. (14) in the available phase space, with $b=1.83 \mathrm{GeV}^{-2}$.

Fragmentation proceeds in an iterative way: String fragments are taken as new strings which are broken again, until the mass of the created fragments is too low to allow further breaking (i.e. projection onto hadrons with the right quantum numbers). Then these final fragments (and those fused strings resulting in the singlet $\{1\}$ representation) are treated as quark clusters and decayed according to combinatorics and phase space. Spin of the produced particles is constructed according to $\mathrm{SU}(2)$ considerations.

The main consequences of string fusion are a strong reduction of multiplicities (both due to the energy-momentum conservation and to the reduction of the effective number of sources of secondaries) and a slight increase of $\left\langle p_{T}^{2}\right\rangle[12]$, an increase in baryon and strangeness production [12, 39], a strong increase in the cumulative effect [45] and a decrease in forward-backward correlations [46].

On the other hand, strings produced in hard collisions (only $g g \longrightarrow g g$ ) are managed by PYTHIA + ARIADNE + JETSET [25, 26" ['. For ARIADNE, PARA(6) is fixed so as the transverse momentum of the radiated gluon should be less than that of the hard gluon (i.e. the one participating in the $g g$ scattering) and $\operatorname{MSTA}(9)=\operatorname{MSTA}(14)=$ $\operatorname{MSTA}(31)=0 . \operatorname{In} \operatorname{JETSET}, \operatorname{PARJ}(41)=1.7 \mathrm{GeV}^{-2}$ and PARJ $(42)=0.6 \mathrm{GeV}^{-2}$; besides, $\operatorname{PARJ}(21)=0.55 \mathrm{GeV} / \mathrm{c}$. Only production of three flavors $(\mathrm{u}, \mathrm{d}, \mathrm{s})$ is considered in the present implementation of the model, so it cannot be used to study production of heavier flavors (some steps in this direction were done in [13]). As a last point, MSTU(4), MSTU(5) and the dimensions in LUJETS have been set to 120000, which has shown to be enough for central $\mathrm{PbPb}$ collisions at LHC. All the other parameters and options in these programs have been set to their default values.

\section{Rescattering of secondaries}

As stated in the Introduction, in this stage the model can be used as an initial condition for further evolution, using either a hydrodynamical model or a microscopic transport as RQMD [16], UrQMD [17, HSD [47], ART [48,... (see [13] for a study of evolution of particle and energy densities). Nevertheless, it is usually assumed that the

\footnotetext{
${ }^{3}$ Although it is not relevant for the generation of momenta of final gluons, in PYTHIA and JETSET the value of $\Lambda_{Q C D}$ has been taken from the corresponding set of parton densities for 5 flavors.
} 
enhancement of hyperons, antihyperons and $\phi$ 's observed in heavy ion experiments at SPS [ : 5, 6, 7, 8] cannot be fully explained by using exclusively a mechanism which goes beyond the independent string hypothesis, as string fusion [12, 16, 17, 18, 39] or baryon junction migration [18, 28, 49, 50, 51]. In order to reproduce these experimental features rescattering of particles in the hadron gas (produced particles among themselves and with spectator nucleons) [52] has been introduced in many models. To tune the code and study nucleus-nucleus collisions, we will make a very simple rescattering model with no space-time evolution, fitted to SPS data. Results of this approach will be presented, but one must keep in mind that predictions which depend critically on rescattering effects should be taken with much care.

Our implementation of rescattering is extremely naif, trying not to solve the full Boltzmann transport equation for all particles but only to make a model, as simple as possible, which gives us an estimation of which effects such rescattering would produce. Neither formation time nor space-time evolution of secondaries are properly considered; instead we require a common minimum density of particles in the rapidity bin of the considered particles, for rescattering to occur. This minimum density, $d N /\left.\left(d y d p_{T}\right)\right|_{\min }=17$, has been chosen for rescattering not to affect results in nucleonnucleon collisions up to the highest energies. Rapidity and $p_{T}$ distances between particles have to be lower than 1.5 units and $0.3 \mathrm{GeV} / \mathrm{c}$ respectively. Only two body reactions have been included, with inverse reactions as required by detailed balance. Spin is ignored, and rescattering takes place before resonance decay. All cross sections are taken equal for all reactions (except for $\Omega$ production and nucleon annihilation).

Operationally, both products of string breaking and spectators are randomly ordered into an array $(1, \ldots, N)$. We compute the possibility of rescattering of the first element with all the others in pairs: $(1,2),(1,3),(1,4), \ldots$ If either in one of the pairs $(1, j), j=2, \ldots, N$, rescattering (either elastic or inelastic) occurs or we reach pair $(1, N)$ with no kind of scattering happening, we go to element 2 and examine the pairs $(2,1),(2,3),(2,4), \ldots$ This is repeated until the pair $(N, N-1)$ is examined. As particles produced in rescattering of the pair $(i, j), i, j=1, \ldots, N, i \neq j$, occupy the same places $i, j$ in the array as their ancestors, particles produced by rescattering have a chance to rescatter again.

The probability for two particles to scatter in a given inelastic channel is $7 \%$ (except for channels involving $\Omega$ 's and nucleon annihilation, where it is 14 and $70 \%$ respectively). For a given process, the probability for elastic scattering is given by 
the sum of those corresponding to all inelastic channels considered for these initial particles. Cross sections (probabilities) are considered energy independent, except for the trivial kinematical thresholds, and isotropic in the center of mass of the colliding secondaries and/or spectators. The considered reactions (together with those for the corresponding antiparticles) [52] can be classified into:

- Light pair, $(q \bar{q})$, annihilation to create another light pair, or light quark exchange: $\pi N \rightarrow \pi N, \pi \pi \rightarrow \pi \pi, \pi Y \rightarrow \pi Y, \pi \Xi \rightarrow \pi \Xi, K N \rightarrow K N$ and $K Y \rightarrow K Y$, where $Y=\Sigma, \Lambda$.

- Other considered reactions are: $\pi N \rightarrow K Y, \pi \pi \rightarrow K \bar{K}, \pi Y \rightarrow K \Xi, \pi \Xi \rightarrow K \Omega$ and $\bar{K} N \rightarrow \phi Y$. These reactions can be classified into:

1. Light pair, $(q \bar{q})$, annihilation to create a $(s \bar{s})$ pair.

2. Reactions with baryon exchange (that is, with three lines in the t-channel).

- Reactions with strangeness exchange: $\bar{K} N \rightarrow \pi Y, \bar{K} Y \rightarrow \pi \Xi, \bar{K} \Xi \rightarrow \pi \Omega$, $K Y \rightarrow \phi N, K \Xi \rightarrow \phi Y$ and $K \Omega \rightarrow \phi Y$. This type of processes can produce (anti)baryons with several strange (anti)quarks and are exothermic.

- Nucleon-antinucleon annihilation into two pions: $N \bar{N} \rightarrow \pi \pi$. This type of reaction has a much larger cross section at low energies than reactions consider before; for this reason its probability has been chosen ten times larger than the others. This is also an effective way to take into account final states involving more than two pions.

To simplify, particles produced in rescattering are always projected onto the lowest spin state. Decay of resonances proceed through the usual JETSET routines, with $\operatorname{MSTJ}(22)=2$, and decay of $\pi^{0}$ 's is forbidden. The results of our rescattering model on strangeness and baryon/antibaryon production can be summarized in three points: hyperon and $\phi$ enhancement, antinucleon annihilation and a slight increase of stopping power (kinematical effects of our rescattering model are very small, due to the applied cuts in rapidity and transverse momentum). Besides, a slight decrease of multiplicities appears, as we will see in the next Section. 


\section{Comparison with experimental data}

In order to show the quality of the choice of the parameters, in this Section we will compare the results of the code with experimental data. We will also analyze the influence of the different physical mechanisms implemented in the model. From now on and unless otherwise stated, results of the code come from its default version with string fusion, rescattering (which do not affect results in nucleon-nucleon collisions, and in pA collisions at SPS energies), and GRV 94 LO [53 parton densities with EKS98 [33] nuclear corrections.

\subsection{Hadron-hadron collisions}

Results of the model for the mean numbers of produced particles in minimum bias pp collisions at $\sqrt{s}=19.4$ and $27.5 \mathrm{GeV}$ are shown in Tables 1 and 2 respectively, compared with experimental data. An overall agreement can be observed. Two comments are in order: On the one hand, the influence of fusion in nucleon-nucleon collisions is tiny, apart from a slight increase in antibaryons. On the other hand, the number of both $\Lambda$ 's and $\bar{\Lambda}$ 's is overestimated in the model. This is due to the fact that in the model, threshold effects, important at these low energies, are treated very roughly (see further comments in the next Subsection). At higher energies the situation improves. For example, in $\bar{p}$ p collisions at $\sqrt{s}=200 \mathrm{GeV}$, the mean number of $\Lambda+\bar{\Lambda}$ in the model is 0.56 , to compare with the experimental result $0.46 \pm 0.12$ [56].

In Fig. 2, rapidity and transverse momentum distributions of negative particles in minimum bias pp collisions at $\sqrt{s}=19.4 \mathrm{GeV}$ are shown and compared with experimental data. The result is satisfactory. In Figs. 3 and 4 pseudorapidity and transverse momentum distributions of charged particles in $\bar{p} p$ collisions at $\sqrt{s}=200$ and 1800 $\mathrm{GeV}$ are compared with experimental data. The agreement is reasonable, although the multiplicity at $200 \mathrm{GeV}$ seems to be slightly underestimated. The results of the model without semihard component are also shown in these Figs., and they do not describe the $p_{T}$ distributions, which justifies the inclusion of hard collisions. Besides, the results given for different sets of parton distributions, both old [53] and new [61, 62] and lead-

ing order or next-to-leading order, are very similar. This fact may look surprising from a pQCD point of view. The main reason is that the cross sections and the number of inelastic collisions in our model are determined by Eqs. (3), (4), (5) and (6), which are independent of the choice of partonic distributions in PYTHIA (this is not so in other 
models, see e.g. [65]). We also think that the quite high $p_{\text {Tmin }}$ we use in PYTHIA, Eq. (17), and the gluon radiation and fragmentation performed by ARIADNE and JETSET, may have some influence on the fact that no difference is apparently seen in the transverse momentum distributions.

In Fig. 5, the evolution of the mean transverse momentum of charged particles is studied in $\overline{\mathrm{p} p}$ collisions at $\mathrm{Sp} \overline{\mathrm{p}} \mathrm{S}$, versus the center of mass energy and, for different particles, versus central charged multiplicity. The trend of data is reproduced and we find the agreement reasonable (this cannot be achieved without the hard component, as seen in this Figure). In Fig. 6 the topological cross section for charged particles in the central region is examined at different energies for $\bar{p} p$ collisions at $\operatorname{Sp} \bar{p} S$, and the agreement is also reasonable, considering that the model slightly underestimates multiplicities at $200 \mathrm{GeV}$ but correctly reproduces those at $1.8 \mathrm{TeV}$, see Figs. 3 and 4 .

\subsection{Proton-nucleus and nucleus-nucleus collisions}

In Table 3 results of the model in pA collisions are compared with experimental data on negative multiplicities. An overall agreement is obtained. The reduction of multiplicities due to string fusion can be observed.

In Table 4 , mean numbers of produced particles are compared with experimental data, for central SS collisions at SPS energies. The agreement is reasonable. Only the number of both $\Lambda$ 's and $\bar{\Lambda}$ 's in the model is significantly below the experimental data. The number of $\Lambda$ 's is increased by both string fusion and rescattering, while that of $\bar{\Lambda}$ 's is mainly determined by only string fusion (see results in $\mathrm{PbPb}$ below). Anyhow, rescattering is seen to have little effect in SS.

Let us now discuss $\mathrm{PbPb}$ collisions at SPS. In the last year a large excitement has arisen in the heavy ion physics community, related to the possibility of Quark Gluon Plasma (QGP) already been obtained at SPS energies [1]. In particular several signals were mentioned, which point out to the existence of QGP. Putting aside the abnormal $J / \psi$ suppression and the excess of dileptons found, there are three signals related to baryon and strangeness production, namely the large enhancement of the (anti)hyperon yields $(\Lambda, \Xi, \Omega)$ in $\mathrm{PbPb}$ collisions compared to $\mathrm{pPb}$, observed by the WA97 [4] and the NA49 [5] Collaborationsf; the linear increase of the inverse exponential slope of

\footnotetext{
${ }^{4} \mathrm{~A}$ recent reanalysis [6] of $\Xi$ data done by the NA49 Collaboration gives yields at midrapidity which are in much closer agreement to the WA97 [4] results than the previous analysis of NA49 [5].
} 
the $m_{T}$ distributions ('temperature') in $\mathrm{PbPb}$ collisions with the mass of the observed particle, except for $\Omega$ [5, 70]; and the different behavior of the temperature between pp and AA collisions. These characteristics have been interpreted as the existence of an intrinsic freeze-out temperature and a collective hydrodynamical flow which is gradually developed: firstly, for $\mathrm{SS}$ collisions, and, in a more clear way, in $\mathrm{PbPb}$ collisions. In this Subsection we will examine some of these points using our model, together with other interesting aspects as $\phi$ production [7, 8], different particle ratios [71] and stopping power [72],

In Fig. 7 we show our results for $\Omega, \Xi$ and $\Lambda$ yields for $\mathrm{pPb}$, and central $\mathrm{PbPb}$ collisions at SPS with four different centralities, together with the experimental data. In order to disentangle the different processes contributing, in Fig. 8 it is shown the results of the code for central $(b \leq 3.2 \mathrm{fm}) \mathrm{PbPb}$ collisions without string fusion and rescattering, with string fusion, and with string fusion and rescattering. A reasonable agreement with data for $\mathrm{PbPb}$ is obtained, only the $\Omega$ 's are a $40 \%$ below the data and we have some excess of $\bar{\Lambda}$ and $\bar{\Xi}^{+}$, see next paragraph. Similar results have been obtained in the Relativistic Quantum Molecular Dynamics model [16, 10] by a mechanism of color ropes which consider fusion of strings; also in the Ultra Relativistic Quantum Molecular Dynamics model [17] and in the HIJING model [18] by using an ad hoc multiplicative factor in the string tension. Also the Dual Parton Model [14, considering the possibility of creation of diquark-antidiquark pairs in the nucleon sea, together with the inclusion of diagrams which take into account baryon junction migration [49, 50, 51, can reproduce the experimental data (for $\Omega$ 's some rescattering has still to be added). The string fusion is the main ingredient to obtain an enhancement of $\bar{\Lambda}$ production and also to reproduce the $\Xi$ data. However rescattering seems fundamental to get enough $\Omega$ 's.

Nevertheless, our results for $\mathrm{pPb}$ are higher than the data for $\bar{\Xi}^{+}$and $\bar{\Lambda}$; this last feature looks quite strange, as $\Lambda$ and $\Xi^{-}$yields agree with data, but we overestimate both $\Lambda$ and $\bar{\Lambda}$ production in pp collisions at this energy plays a minor role in minimum bias $\mathrm{pPb}$ collisions, this turns out to be a result of string fusion. About $\bar{\Lambda}$, our results are higher than the WA97 data also in $\mathrm{PbPb}$, its pro-

\footnotetext{
${ }^{5}$ In our opinion, the comparison of (anti)hyperon nucleus-nucleus data with those in nucleonnucleon collisions should be taken with caution at SPS, because at this relatively low energy the nucleon-nucleon value rises sharply with increasing energy due to the $t_{m i n^{-}}$and delayed threshold effects [73], which usually are not properly implemented in models.
} 
duction being mainly determined by string fusion and hardly affected by rescattering. This fact makes that our results for $\mathrm{PbPb}$ are really an extrapolation in the model from the value for $\bar{\Lambda}$ production in central SS collisions by the NA35 Collaboration, which was used to fix the fusion cross section $\sigma_{\text {fus }}$ [39] (even so, the model underestimates $\bar{\Lambda}$ production in central SS, see Table 4). So, from the point of view of our model, there exists either a large $\bar{\Lambda}$ annihilation or a conflict between NA35 data for SS and WA97 data for $\mathrm{PbPb}$ and $\mathrm{pPb}$.

In Fig. 9 we plot the inverse exponential slopes of the $m_{T}$ distributions for different particles, together with the WA97 experimental dataf. A semiquantitative agreement is obtained. In particular it can be seen that the $\Omega$ slope does not obey the linear increase with increasing mass both in the model and in data, and that rescattering slightly increases temperatures.

About $\phi$ enhancement, our integrated yields per event without fusion, with fusion, and with fusion and rescattering are 3.55, 4.20 and 5.35 respectively, in rough agreement with experimental data, $7.6 \pm 1.1$ [8]. In Fig. 10 the stopping power is shown, i.e. the $p-\bar{p}$ rapidity distributions for central $\mathrm{PbPb}$ collisions at SPS, compared with the experimental data $[72]$, together with the predictions for RHIC and LHC energies. This quantity is essentially determined by the string fusion mechanism and rescattering only plays a minor role. As discussed for strangeness enhancement, it has been pointed out that baryon junction migration [49, 50, 51] will enhance the stopping power due to diagrams additional to the usual ones of the Dual Parton Model. The inclusion of these diagrams also explains the SPS data. We have not taken into account such diagrams to avoid double counting, because in the fusion of strings they are partially included in an effective way. In Fig. 11 the antiproton rapidity distribution in central $\mathrm{PbPb}$ collisions is presented and compared to the experimental data [74; a great suppression of the antiproton yield is seen, due to rescattering.

In Table 5 our results for the ratios between different particles are compared with the experimental data 71$]$ for PbPb central collisions at SPS. We observe an overall, rough agreement with the SPS data, with some excess of $\bar{\Lambda}$ and $\bar{\Xi}^{+}$, see Fig. 7 and comments above.

Let us emphasize that we obtain a semiquantitative agreement with the experimen-

\footnotetext{
${ }^{6}$ The fits have been performed in the same $m_{T}$ regions as WA97 did 70 . For statistical reasons, we compare the slopes in the model for yields integrated over all rapidities, with experimental data taken in the central rapidity region.
} 
tal data in $\mathrm{PbPb}$, in three of the features advocated as signals of QGP production. We are only below data in $\Omega$ production by less than a factor 2 . So we think that our rescattering model, being very simple, can be useful as a tool to show the trend of such effect and at least help to tune the initial condition which can be used in transport models.

Finally, let us comment on multiplicities in $\mathrm{PbPb}$ collisions at SPS energies. For a centrality of $5 \%$ (corresponding in the model to $b \leq 3.4 \mathrm{fm}$ ), we get, for $d N^{-} / d y$ at $y=0,265,250$ and 235 without string fusion, with string fusion, and with string fusion and rescattering respectively. Experimentally, the NA49 Collaboration gets $196 \pm 10$ [75], while the WA97 Collaboration gets $178 \pm 22$ [76]. In view of these data the code overestimates multiplicities. On the other hand, if we compare the charged multiplicity per participant (wounded) nucleon and pseudorapidity unit at midrapidity versus the number of wounded nucleons in $\mathrm{PbPb}$ collisions at SPS, with data from the WA98 Collaboration [77], the trend of data seems to be reproduced, while their magnitude is underestimated [78. In Fig. 12 we show the rapidity distribution of negatives compared with NA49 data 775 .

\section{Predictions for RHIC and LHC}

Predictions for pseudorapidity and transverse momentum distributions of charged particles in nucleon-nucleon and central nucleus-nucleus collisions at $\operatorname{RHIC}(\sqrt{s}=200$ GeV per nucleon) and LHC ( $\sqrt{s}=5.5$ and $14 \mathrm{TeV}$ per nucleon for nucleus-nucleus and nucleon-nucleon collisions respectively) can be seen for nucleus-nucleus and nucleonnucleon collisions respectively) in Figs. 13, 14 and 15. While at SPS the influence of string fusion on multiplicities at midrapidity is of the order $10 \div 15 \%$, at RHIC it reaches a $30 \div 35 \%$. In these Figures, the large influence of the hard contribution at LHC can be observed. Again, the striking fact of the small influence of parton densities appears, both in nucleon and in nuclear collisions. On the other hand, the scaling of nucleon-nucleon with the number of wounded nucleons (the Wounded Nucleon Model [79) gives predictions which lie far below any of those of our model.

In Fig. 9 we plot the inverse exponential slopes of the $m_{T}$ distributions for different particles at RHIC. We see that, compared to the SPS situation, temperatures get higher

\footnotetext{
${ }^{7}$ About the reliability of predictions for RHIC and LHC, see comments in the last paragraph of Subsection 2.3 and in the first paragraph of Section 4.
} 
in all cases, as expected.

We present our predictions for different particle ratios at RHIC and LHC in Table 6. It can be observed that our results are not very different to those of statistical models [10, 80, 81, 82. However, strangeness enhancement in our case has nothing to do with thermal and/or chemical equilibrium. The main difference in the predictions for RHIC and LHC between the String Fusion Model and statistical models is the overall charged multiplicity, which is respectively 950 and 3100 for SFM and 1500 and 7600 for statistical models [11] (assuming initial temperatures of 500 and $1000 \mathrm{MeV}$ for RHIC and LHC respectively). Besides, predictions for the stopping power at RHIC and LHC energies are presented in Fig. 10. Now, a pronounced dip appears at midrapidities.

Detailed discussions on first RHIC results will be given elsewhere [78. Here we simply compare our results with some preliminary data of the PHOBOS [19] and PHENIX 20] Collaborations at RHIC. For charged particles we obtain $d N /\left.d \eta\right|_{|\eta|<1}=520$ and 585 for the $6 \%$ more central AuAu collisions at $\sqrt{s}=56$ and $130 \mathrm{GeV}$ per nucleon respectively, to be compared with $408 \pm 12$ (stat.) \pm 30 (syst.) and $555 \pm 12$ (stat.) \pm 35 (syst.) $(609 \pm 1$ (stat.) \pm 37 (syst.)) in PHOBOS (PHENIX). Our prediction for $\sqrt{s}=200$ $\mathrm{GeV}$ per nucleon with the same centrality cut is $d N /\left.d \eta\right|_{|\eta|<1}=635$.

\section{Conclusions}

A Monte Carlo model for nucleon and nuclear collisions in the energy range going from SPS to LHC has been presented. It is based on a partonic realization of Regge-Gribov and Glauber-Gribov models and its translation to strings following the DPM/QGSM ideas. A hard component is included to reproduce the high transverse momentum tail of the spectrum. Collectivity is included considering the possibility of fusion of pairs of strings. Strings are decayed in a conventional way. In order to tune the parameters of the model and apply it to collisions between nuclei, a naif model of rescattering has been introduced.

The results of the models turn out to agree reasonably with total multiplicities, and longitudinal and transverse momentum spectra in the energy range from SPS to

\footnotetext{
${ }^{8}$ The code, called psm-1.0, has been written in Fortran 77 and can be taken as an uuencoded file, containing instructions for installation and use, by anonymous ftp from ftp://ftp.uco.es/pub/fa1arpen/, or from the web sites http://www.uco.es/fa1arpen/ or http://fpaxp1.usc.es/phenom/, or requested from the authors.
} 
TeVatron. The agreement with strangeness production, temperature behavior and stopping at SPS is semiquantitative.

There exist other Monte Carlo models for multiparticle production in nuclear collisions at ultrarelativistic energies (see [11 for a review): RQMD [16], UrQMD [17, HIJING [27], DPMJET [28], HSD [47], NEXUS [83], VNI [84, AMPT [85], LUCIFER [86, ,... Let us examine the main similarities and differences, concerning the stage before rescattering is applied. Both DPMJET and our model are realizations of the DPM/QGSM which include a hard component, but we introduce string fusion, while DPMJET considers diquark breaking diagrams. RQMD takes into account string fusion (and now UrQMD and HIJING [18] in a simple way), but no hard part is included either in RQMD or in UrQMD. The main difference with HIJING lies in the soft component, which is considered energy independent is HIJING (and in this way, the multiplicity increase with increasing energy is mainly due to the hard component), while in our case it increases as an unitarized supercritical pomeron. VNI is a parton cascade code, in which the initial stage is mainly generated by hard collisions, with no hadronic degrees of freedom (strings). AMPT is a hybrid code, which uses HIJING as initial condition for parton cascade and, after hadronization, performs hadronic transport. HSD is focused in the transport of hadronic degrees of freedom, the initial stage not coming from strings stretched between partons of projectile and target, but considering strings as excitations of nucleons in the projectile and target, as in Fritiof [87]; similar comments can be made for LUCIFER. Finally, NEXUS is based in DPM/QGSM, trying to solve the problem of energy-momentum conservation for both cross sections and multiparticle amplitudes at the same time. In our model, energy conservation is strictly taken into account only for multiparticle amplitudes. Besides NEXUS takes into account triple pomeron diagrams, which in our case are effectively included in string fusion.

A detailed comparison of results of the model with the first RHIC results will be presented elsewhere 78. As future developments, strangeness production should be reconsidered and production of heavier flavors included. Also fusion of more than two strings and the possibility of a phase transition like percolation of strings is needed in order to improve predictions for LHC and study the possibility of QGP formation in the framework of string models. 
Acknowledgments: We thank M. A. Braun and E. G. Ferreiro, who participated in early stages of this work. We also thank G. S. Bali, A. Capella, K. J. Eskola, A. B. Kaidalov, C. A. Salgado, Yu. M. Shabelski and K. Werner for useful discussions, and J. Stachel for comments on the predictions of the statistical models for RHIC and LHC. N. A. and C. P. acknowledge financial support by CICYT of Spain under contract AEN99-0589-C02 and N. S. A. by Academy of Finland under grant number 48477. N. A. and D. S. also thank Universidad de Córdoba and Fundación Barrié de la Maza of Spain respectively, for financial support. N. A. thanks Departamento de Física de Partículas of the Universidade de Santiago de Compostela, and D. S. the ALICE Collaboration at CERN, for hospitality during stays in which part of this work was completed. Laboratoire de Physique Théorique is Unité Mixte de Recherche - CNRS - UMR n 8627. 


\section{References}

[1] CERN Press Release, February 10th 2000; U. Heinz and M. Jacob, nuclth/0002042.

[2] M. Gyulassy, Prog. Theor. Phys. Suppl. 140 (2000) 68; D. Zschiesche et al., nuclth/0101047.

[3] NA50 Collaboration: M. C. Abreu et al., Phys. Lett. B410 (1997) 327; ibid. 337.

[4] WA97 Collaboration: E. Andersen et al., Phys. Lett. B433 (1998) 209; ibid. B449 (1999) 401.

[5] NA49 Collaboration: H. Appelshäuser et al., Phys. Lett. B444 (1998) 523; Eur. Phys. J. C2 (1998) 661.

[6] NA49 Collaboration: R. A. Barton et al., in Proceedings of the Strangeness 2000 Conference (Berkeley, USA, July 20th-25th 2000).

[7] NA50 Collaboration: N. Willis et al., Nucl. Phys. A661 (1999) 534c.

[8] NA49 Collaboration: G. Höhne et al., Nucl. Phys. A661 (1999) 485c; S. V. Afanasev et al., Phys. Lett. B491 (2000) 59.

[9] CERES Collaboration: G. Agakichiev et al., Phys. Rev. Lett. 75 (1995) 1272; Phys. Lett. B422 (1998) 405.

[10] S. A. Bass et al., Nucl. Phys. A661 (1999) 205c.

[11] N. Armesto and C. Pajares, Int. J. Mod. Phys. A15 (2000) 2019.

[12] N. S. Amelin, M. A. Braun and C. Pajares, Phys. Lett. B306 (1993) 312; Z. Phys. C63 (1994) 507.

[13] N. S. Amelin, H. Stöcker, W. Greiner, N. Armesto, M. A. Braun and C. Pajares, Phys. Rev. C52 (1995) 362.

[14] A. Capella, U. P. Sukhatme, C.-I. Tan and J. Tran Thanh Van, Phys. Lett. 81B (1979) 69; Phys. Rept. 236 (1994) 225.

[15] A. B. Kaidalov and K. A. Ter-Martirosyan, Phys. Lett. B117 (1982) 247. 
[16] H. Sorge, H. Stöcker and W. Greiner, Ann. Phys. 192 (1989) 266; H. Sorge, M. Berenguer, H. Stöcker and W. Greiner, Phys. Lett. B289 (1992) 6; H. Sorge, Phys. Rev. C52 (1995) 3291.

[17] S. Soff, S. A. Bass, M. Bleicher, L. Bravina, E. Zabrodin, H. Stöcker and W. Greiner, Phys. Lett. B471 (1999) 89; M. Bleicher, M. Belkacem, S. A. Bass, S. Soff and H. Stöcker, Phys. Lett. B485 (2000) 213; M. Bleicher, W. Greiner, H. Stöcker and N. Xu, Phys. Rev. C62 (2000) 061901.

[18] S. E. Vance, in Proceedings of the Strangeness 2000 Conference (Berkeley, USA, July 20th-25th 2000), nucl-th/0012056.

[19] PHOBOS Collaboration: B. B. Back et al., Phys. Rev. Lett. 85 (2000) 3100.

[20] PHENIX Collaboration: K. Adcox et al., nucl-ex/0012008.

[21] L. R. B. Elton, Nuclear sizes, Oxford University Press, Oxford 1961.

[22] A. DeShalit and H. Feshbach, Theoretical Nuclear Physics, Vol. 1: Nuclear Structure, John Wiley \& Sons, New York 1974.

[23] V. A. Abramovski, E. V. Gedalin. E. G. Gurvich and O. V. Kancheli, Sov. J. Nucl. Phys. 53 (1991) 172.

[24] A. B. Kaidalov, Sov. J. Nucl. Phys. 45 (1987) 902; Yu. M. Shabelski, Z. Phys. C57 (1993) 409.

[25] T. Sjöstrand, Comput. Phys. Commun. 82 (1994) 74.

[26] L. Lönnblad, Comput. Phys. Commun. 71 (1992) 15.

[27] X.-N. Wang and M. Gyulassy, Comput. Phys. Commun. 83 (1994) 307.

[28] J. Ranft, preprint SI-99-5 (hep-ph/9911213); preprint SI-99-6 (hep-ph/9911232);

S. Roesler, R. Engel and J. Ranft, preprint SLAC-PUB-8740 (hep-ph/0012252).

[29] J.-P. Blaizot and A. H. Mueller, Nucl. Phys. B289 (1987) 847; L. V. Gribov, E. M. Levin and M. G. Ryskin, Phys. Rept. 100 (1983) 1; A. H. Mueller and J.-W. Qiu, Nucl. Phys. B268 (1986) 427; J. Jalilian-Marian, A. Kovner, L. McLerran and H. Weigert, Phys. Rev. D55 (1997) 5414; A. H. Mueller, Nucl. Phys. B558 (1999) 285. 
[30] K. J. Eskola, K. Kajantie, P. V. Ruuskanen and K. Tuominen, Nucl. Phys. B570 (2000) 379; K. J. Eskola, K. Kajantie and K. Tuominen, Phys. Lett. B497 (2001) 39; D. Kharzeev and M. Nardi, nucl-th/0012025.

[31] Particle Data Group: D. E. Groom et al., Eur. Phys. J. C15 (2000) 1.

[32] H. Plothow-Besch, PDFLIB Version 8.04: User's Manual, CERN Program Library Entry W5051 PDFLIB (2000).

[33] K. J. Eskola, V. J. Kolhinen and P. V. Ruuskanen, Nucl. Phys. B535 (1998) 351; K. J. Eskola, V. J. Kolhinen and C. A. Salgado, Eur. Phys. J. C9 (1999) 61.

[34] K. J. Eskola, J. Qiu and J. Czyzewski, private communication; V. Emel'yanov, A. Khodinov, S. R. Klein and R. Vogt, Phys. Rev. C56 (1997) 2726.

[35] A. Capella, E. G. Ferreiro and A. B. Kaidalov, Eur. Phys. J. C11 (1999) 163.

[36] N. Armesto, M. A. Braun, E. G. Ferreiro and C. Pajares, Phys. Rev. Lett. 77 (1996) 3736 .

[37] M. Nardi and H. Satz, Phys. Lett. B442 (1998) 14; H. Satz, Nucl. Phys. A642 (1998) 130; J. Dias de Deus, R. Ugoccioni and A. Rodrigues, Eur. Phys. J. C16 (2000) 537.

[38] M. A. Braun, C. Pajares and J. Ranft, Int. J. Mod. Phys. A14 (1999) 2689.

[39] N. Armesto, M. A. Braun, E. G. Ferreiro and C. Pajares, Phys. Lett. B344 (1995) 301; E. G. Ferreiro, C. Pajares and D. Sousa, Phys. Lett. B422 (1998) 314.

[40] J. Schwinger, Phys. Rev. 82 (1951) 664; E. Brezin and C. Itzykson, Phys. Rev. D2 (1970) 1191.

[41] G. S. Bali, Phys. Rev. D62 (2000) 114503; preprint HUB-EP-99-67 hepph/0001312).

[42] Yu. A. Simonov, JETP Lett. 71 (2000) 127; V. I. Shevchenko and Yu. A. Simonov, Phys. Rev. Lett. 85 (2000) 1811.

[43] X. Artru and G. Mennessier, Nucl. Phys. B70 (1974) 93; X. Artru, Phys. Rept. 97 (1983) 147. 
[44] B. Andersson, G. Gustafson, G. Ingelman and T. Sjöstrand, Phys. Rept. 97 (1983) 31.

[45] N. Armesto, M. A. Braun, E. G. Ferreiro, C. Pajares and Yu. M. Shabelski, Phys. Lett. B389 (1996) 78; Astropart. Phys. 6 (1997) 327; N. Armesto, E. G. Ferreiro, C. Pajares and Yu. M. Shabelski, Z. Phys. C73 (1997) 309.

[46] N. S. Amelin, N. Armesto, M. A. Braun, E. G. Ferreiro and C. Pajares, Phys. Rev. Lett. 73 (1994) 2813.

[47] W. Cassing and E. L. Bratkovskaya, Phys. Rept. 308 (1999) 65; Nucl. Phys. A623 (1997) 570 .

[48] B.-A. Li and C. M. Ko, Phys. Rev. C52 (1995) 2037.

[49] A. Capella and C. A. Salgado, Phys. Rev. C60 (1999) 054906; preprint LPTORSAY-00-66 (hep-ph/0007236); A. Capella, E. G. Ferreiro and C. A. Salgado, Phys. Lett. B459 (1999) 27.

[50] S. E. Vance and M. Gyulassy, Phys. Rev. Lett. 83 (1999) 1735.

[51] A. Capella and B. Z. Kopeliovich, Phys. Lett. B381 (1996) 325; B. Z. Kopeliovich and B. Povh, Phys. Lett. B446 (1999) 321; D. Kharzeev, Phys. Lett. B378 (1996) 238; F. W. Bopp, hep-ph/0002190.

[52] P. Koch, B. Müller and J. Rafelski, Phys. Rept. 142 (1986) 167.

[53] M. Glück, E. Reya and A. Vogt, Z. Phys. C67 (1995) 433.

[54] M. Gazdzicki and H. Hansen, Nucl. Phys. A528 (1991) 754; H. Bialkowska, M. Gazdzicki, W. Retyk and E. Skrzypczak, Z. Phys C55 (1992) 491.

[55] LEBC-EHS Collaboration: M. Aguilar-Benítez et al., Z. Phys. C50 (1991) 405.

[56] UA5 Collaboration: R. E. Ansorge et al., Nucl. Phys. B328 (1989) 36.

[57] NA5 Collaboration: C. De Marzo et al., Phys. Rev. D26 (1982) 1019.

[58] NA35 Collaboration: H. Strobele et al., Z. Phys. C38 (1988) 89.

[59] UA5 Collaboration: G. J. Alner et al., Z. Phys. C33 (1986) 1. 
[60] UA1 Collaboration: C. Albajar et al., Nucl. Phys. B335 (1990) 261.

[61] H. L. Lai et al., Eur. Phys. J. C12 (2000) 375.

[62] M. Glück, E. Reya and A. Vogt, Eur. Phys. J. C5 (1998) 461.

[63] CDF Collaboration: F. Abe et al., Phys. Rev. D41 (1990) 2330.

[64] CDF Collaboration: F. Abe et al., Phys. Rev. Lett. 61 (1988) 1819.

[65] D. Petermann, J. Ranft and F. W. Bopp, Z. Phys. C54 (1992) 685.

[66] E735 Collaboration: T. Alexopoulos et al., Phys. Rev. D48 (1993) 984.

[67] NA35 Collaboration: J. Bartke et al., Z. Phys. C48 (1990) 191.

[68] E154 Collaboration: D. H. Brick et al., Phys. Rev. D39 (1989) 2484.

[69] NA35 Collaboration: T. Alber et al., Eur. Phys. J. C2 (1998) 643.

[70] WA97 Collaboration: F. Antinori et al., Nucl. Phys. A661 (1999) 481c; Eur. Phys. J. C14 (2000) 633.

[71] WA97 Collaboration: I. Králik et al., Nucl. Phys. A638 (1998) 115c.

[72] NA49 Collaboration: G. E. Cooper et al., Nucl. Phys. A661 (1999) 362c.

[73] A. Capella, U. P. Sukhatme, C.-I. Tan and J. Tran Thanh Van, Phys. Rev. D36 (1987) 109 .

[74] NA49 Collaboration: J. Bächler et al., Nucl. Phys. A661 (1999) 45c.

[75] NA49 Collaboration: H. Appelshäuser et al., Phys. Rev. Lett. 82 (1999) 2471; P. G. Jones et al., Nucl. Phys. A610 (1996) 188c.

[76] WA97 Collaboration: F. Antinori et al., Nucl. Phys. A661 (1999) 130c.

[77] WA98 Collaboration: M. M. Aggarwal et al., nucl-ex/0008004.

[78] N. Armesto, C. Pajares and D. Sousa, in preparation.

[79] A. Bialas, M. Bleszyński and W. Czyz, Nucl. Phys. B111 (1976) 461; A. Bialas, in Proceedings of the XIIIth International Symposium on Multiparticle Dynamics, ed. W. Kittel, W. Metzger and A. Stergiou (World Scientific, Singapore, 1983). 
[80] P. Braun-Munzinger, I. Heppe and J. Stachel, Phys. Lett. B465 (1999) 15.

[81] P. Braun-Munzinger, Nucl. Phys. A661 (1999) 261c.

[82] J. Stachel, in Proceedings of the XXIXth International Symposium on Multiparticle Dynamics (Providence, USA, August 9th-13th 1999), to be published by World Scientific.

[83] H. J. Drescher, M. Hladik, K. Werner and S. Ostapchenko, Nucl. Phys. Proc. Suppl. 75A (1999) 275; H. J. Drescher, M. Hladik, S. Ostapchenko, T. Pierog and K. Werner, preprint SUBATECH-00-07 (hep-ph/0006247); preprint SUBATECH00-06 (hep-ph/0007198).

[84] K. Geiger and B. Müller, Nucl. Phys. B369 (1992) 600; K. Geiger, Phys. Rev. D47 (1993) 133; Comput. Phys. Commun. 104 (1997) 70.

[85] B. Zhang, C. M. Ko, B.-A. Li and Z. Lin, Phys. Rev. C61 (2000) 067901; Z. Lin, S. Pal, C. M. Ko, B.-A. Li and B. Zhang, nucl-th/0011059.

[86] D. E. Kahana and S. H. Kahana, Phys. Rev. C58 (1998) 3574; ibid. C59 (1999) $1651 ;$ nucl-th/0010043.

[87] B. Andersson, G. Gustafson and B. Nilsson-Almqvist, Nucl. Phys. B281 (1987) 289. 


\section{List of figures:}

1. Upper plot: Results in the model for the total (solid line), production (dashed line) and hard (dotted line) cross sections versus $\sqrt{s}$, compared with experimental data for total cross sections in pp (filled circles) and $\bar{p}$ p (open circles) collisions taken from [31]. Lower plot: $p_{\text {Tmin }}$ (solid line) used in the model, and model results for the mean number of total (dashed line) and hard (dotted line) inelastic collisions per event versus $\sqrt{s}$ computed for the same collisions as in the upper plot. The ordinary reggeon contribution, which decreases quickly with increasing energy, is not included, see text.

2. Results of the code for the rapidity distribution (upper plot), and the $p_{T}$ distribution for particles with $2<y_{l a b}<4$ (lower plot), of negative particles in minimum bias pp collisions at $p_{l a b}=200 \mathrm{GeV} / \mathrm{c}$, compared with experimental data [57, 58].

3. Results of the code for the pseudorapidity distribution (upper plot), and the $p_{T}$ distribution for particles with $|\eta|<2.5$ (lower plot), of charged particles in minimum bias $\overline{\mathrm{p} p}$ collisions at $\sqrt{s}=200 \mathrm{GeV}$, compared with experimental data [59, 60]. Solid lines are the results with GRV 94 LO parton densities [53], dashed lines with CTEQ5L [61, dotted lines with GRV $98 \mathrm{HO}$ 62 and dashed-dotted lines results without semihard contribution. In the $p_{T}$ distribution, model results have been normalized to experimental data.

4. Results of the code for the pseudorapidity distribution (upper plot), and the $p_{T}$ distribution for particles with $|\eta|<1$ (lower plot), of charged particles in minimum bias $\bar{p}$ p collisions at $\sqrt{s}=1.8 \mathrm{TeV}$, compared with experimental data [63, 64]. Line convention is the same as in Fig. 3. In the $p_{T}$ distribution, model results have been normalized to experimental data.

5. Upper plot: results of the code without hard part (dotted line), without string fusion (dashed line) and with string fusion (solid line) for $\left\langle p_{T}\right\rangle$ of charged particles with $|\eta|<$ 0.5 in $\bar{p}$ p collisions, versus $\sqrt{s}$, compared with UA1 data [60] and a parametrization given in this reference (dashed-dotted line). Lower plot: results of the code for $\left\langle p_{T}\right\rangle$ of $\pi^{ \pm}$(solid line), $K^{ \pm}$(dashed line) and $\overline{\mathrm{p}}$ (dotted line) in $\overline{\mathrm{p}} \mathrm{p}$ collisions at $\sqrt{s}=1.8 \mathrm{TeV}$ versus the pseudorapidity density of charged particles for $|\eta|<3.15$, compared with 
E735 data 66] for $\pi^{ \pm}$(circles), $K^{ \pm}$(squares) and $\overline{\mathrm{p}}$ (triangles).

6. Results in the model (solid lines, arbitrarily normalized) for topological cross sections of charged particles with $|\eta|<2.5$ in $\bar{p}$ p collisions, compared with experimental data from [60]. Upper curves and data correspond to $\sqrt{s}=0.9 \mathrm{TeV}$, those in the middle (multiplied by 0.1 ) to $\sqrt{s}=0.5 \mathrm{TeV}$, and lower curves and data (multiplied by $0.01)$ to $\sqrt{s}=0.2 \mathrm{TeV}$.

7. Yields per unity of rapidity at central rapidity, as a function of the number of wounded nucleons, for $\Lambda, \Xi^{-}$and $\Omega^{-}+\bar{\Omega}^{+}$(left), and for $\bar{p}, \bar{\Lambda}$ and $\bar{\Xi}^{+}$(right), for $\mathrm{pPb}$ collisions and four different centralities in PbPb collisions at SPS energies. Full lines represent our calculation with string fusion, and dashed lines with fusion and rescattering. Experimental data are from the WA97 Collaboration [4].

8. Results in the model (dotted line: without fusion, dashed line: with fusion, solid line: with fusion and rescattering) for strange baryon production in central $\mathrm{PbPb}$ collisions (5\% centrality) at SPS compared with experimental data from the WA97 Collaboration [4] (triangles) and the NA49 Collaboration [5] (squares).

9. Results in the model (filled circles: with fusion, open circles: with fusion and rescattering) for the inverse exponential slope of the $m_{T}$ distributions at midrapidity of different particles versus the mass of the particles in central (5\% centrality) $\mathrm{PbPb}$ collisions at SPS, compared with the experimental data of the WA97 Collaboration 70 (3.5\% centrality, open squares). We also present our predictions for the same collisions at RHIC energy with fusion, filled triangles, and with fusion and rescattering, open triangles.

10. Results in the model for the $p-\bar{p}$ rapidity distribution in central ( $5 \%$ centrality) $\mathrm{PbPb}$ collisions at SPS (a), solid line), and RHIC and LHC (b)), dashed and dotted lines respectively), compared with experimental data at SPS [72].

11. Results in the model (dotted line: without fusion, solid line: with fusion, dashed line: with fusion and rescattering) for the $\bar{p}$ rapidity distribution in central (5\% centrality) $\mathrm{PbPb}$ collisions at SPS, compared with experimental data [74].

12. Results in the model for the rapidity distribution of negative particles in central 
(5\% centrality) $\mathrm{PbPb}$ collisions at $\sqrt{s}=17.3 \mathrm{GeV}$ per nucleon, without fusion (dotted line), with fusion (dashed line) and with fusion and rescattering (solid line), compared with data from NA49 [75].

13. Results of the code for the pseudorapidity distributions (upper plots), and the $p_{T}$ distributions for particles with $|\eta|<2.5$ (lower plots), of charged particles in central $(b \leq 3.2 \mathrm{fm})$ AuAu collisions at $\sqrt{s}=200 \mathrm{GeV}$ per nucleon. In the plots on the left, solid lines are results with EKS98 [33] parametrization of parton densities inside nuclei, dashed lines with a parametrization as $F_{2 A}$ [34], and dotted lines without modification of parton densities inside nuclei. In the plots on the right, solid lines are results without string fusion, dashed lines with string fusion, dotted lines are nucleon-nucleon results at the same energy, scaled by the number of wounded nucleons (344.6/2), and dasheddotted lines are results with string fusion and rescattering.

14. Results of the code for the pseudorapidity distribution (upper plot), and the $p_{T}$ distribution for particles with $|\eta|<2.5$ (lower plot), of charged particles in minimum bias pp collisions at $\sqrt{s}=14000 \mathrm{GeV}$. Line convention is the same as in Fig. 3 (the dashed-dotted line is absent).

15. Results of the code for the pseudorapidity distributions (upper plots), and the $p_{T}$ distributions for particles with $|\eta|<2.5$ (lower plots), of charged particles in central $(b \leq 3.2 \mathrm{fm}) \mathrm{PbPb}$ collisions at $\sqrt{s}=5500 \mathrm{GeV}$ per nucleon. In the plots on the left, line convention is the same as in Fig. 13 left, but results have been obtained without rescattering and the dashed-dotted lines show results without semihard contribution. In the plots on the right, solid lines are results without string fusion, dashed lines with string fusion, dotted lines are nucleon-nucleon results at the same energy, scaled by the number of wounded nucleons $(382.1 / 2)$, and dashed-dotted lines are results with string fusion and rescattering. 


\section{List of tables:}

1. Results in the model for mean multiplicities of different particles in minimum bias pp collisions at $p_{l a b}=200 \mathrm{GeV} / \mathrm{c}$, without and with string fusion, compared with experimental data [54].

2. Results in the model for mean multiplicities of different particles in minimum bias pp collisions at $\sqrt{s}=27.5 \mathrm{GeV}$, without and with string fusion, compared with experimental data 55 .

3. Results in the model for mean multiplicities of negative particles in minimum bias pA collisions at $p_{l a b}=200 \mathrm{GeV} / \mathrm{c}$, without and with string fusion, compared with experimental data for pS [67, pAr and pXe [57, and pAg and pAu 668.

4. Results in the model for mean multiplicities of different particles in central $(b \leq 1.3$ fm) SS collisions at $\sqrt{s}=19.4 \mathrm{GeV}$ per nucleon, compared with experimental data [69]. Results are presented without fusion (NF), with fusion (F), and with fusion and rescattering (FR).

5. Results in the model for different particle ratios at midrapidity in central $(30 \%$ centrality) $\mathrm{PbPb}$ collisions at SPS, compared with experimental data [71], following the same convention as in Table 4 .

6. Results in the model for different particle ratios at midrapidity in central $(b \leq 3.2$ fm) AuAu collisions at RHIC and PbPb collisions at LHC, following the same convention as in Table 4 . For comparison, results from other models (Quark Coalescence Model (QCM) [10], Rafelski [10] and B-M [10, 80]) for RHIC are included. 
Figures:
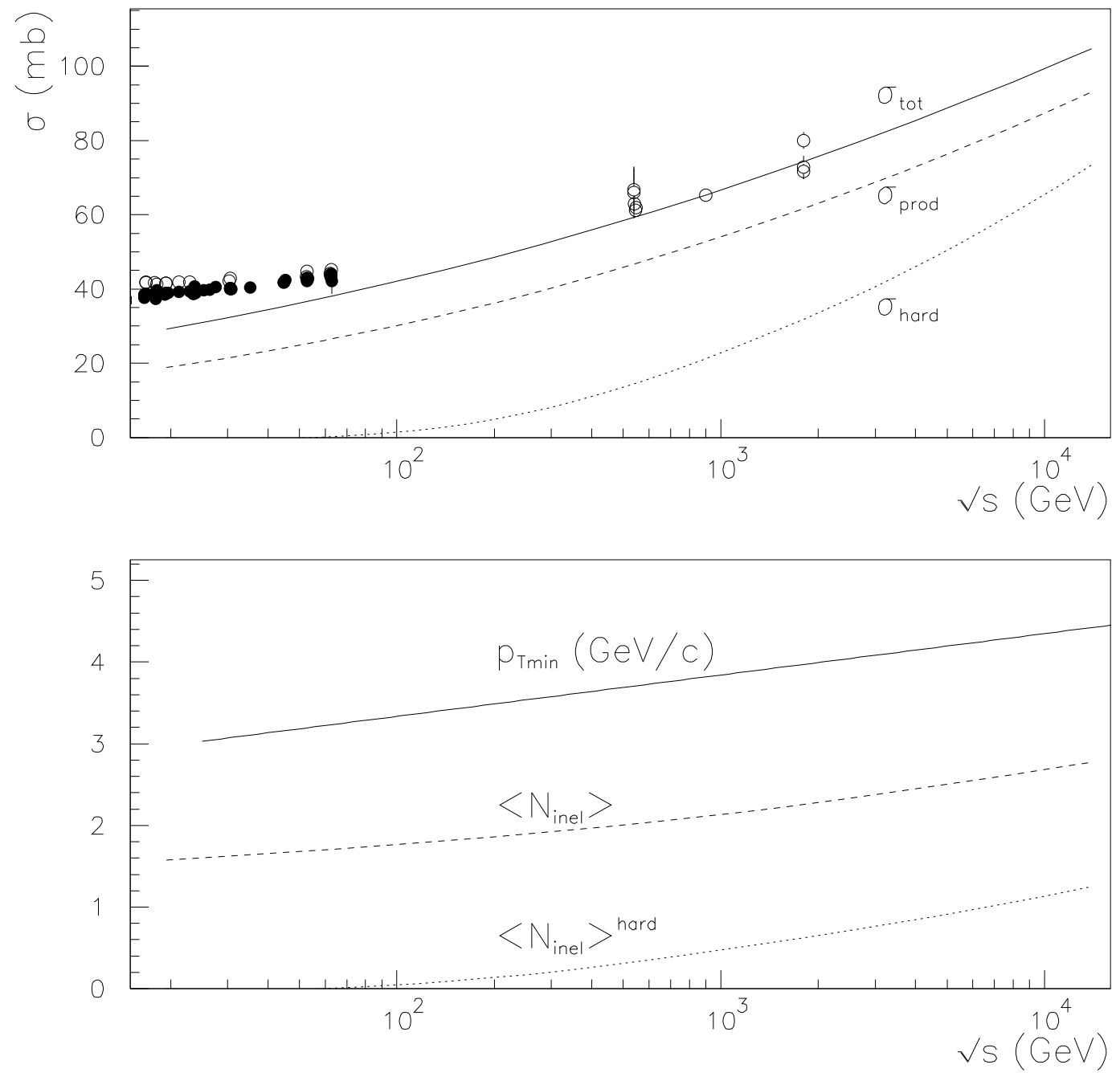

Figure 1: 

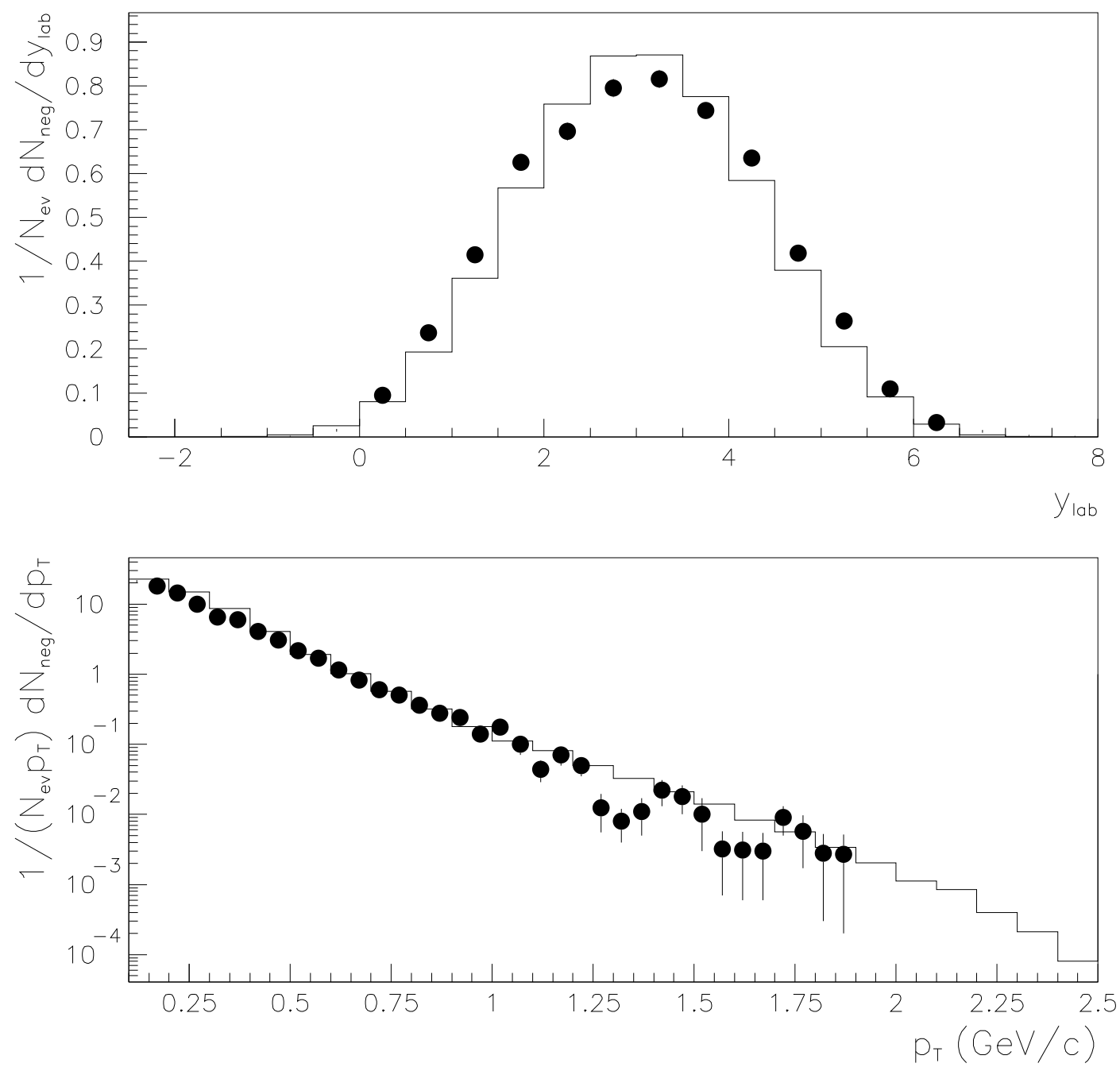

Figure 2: 

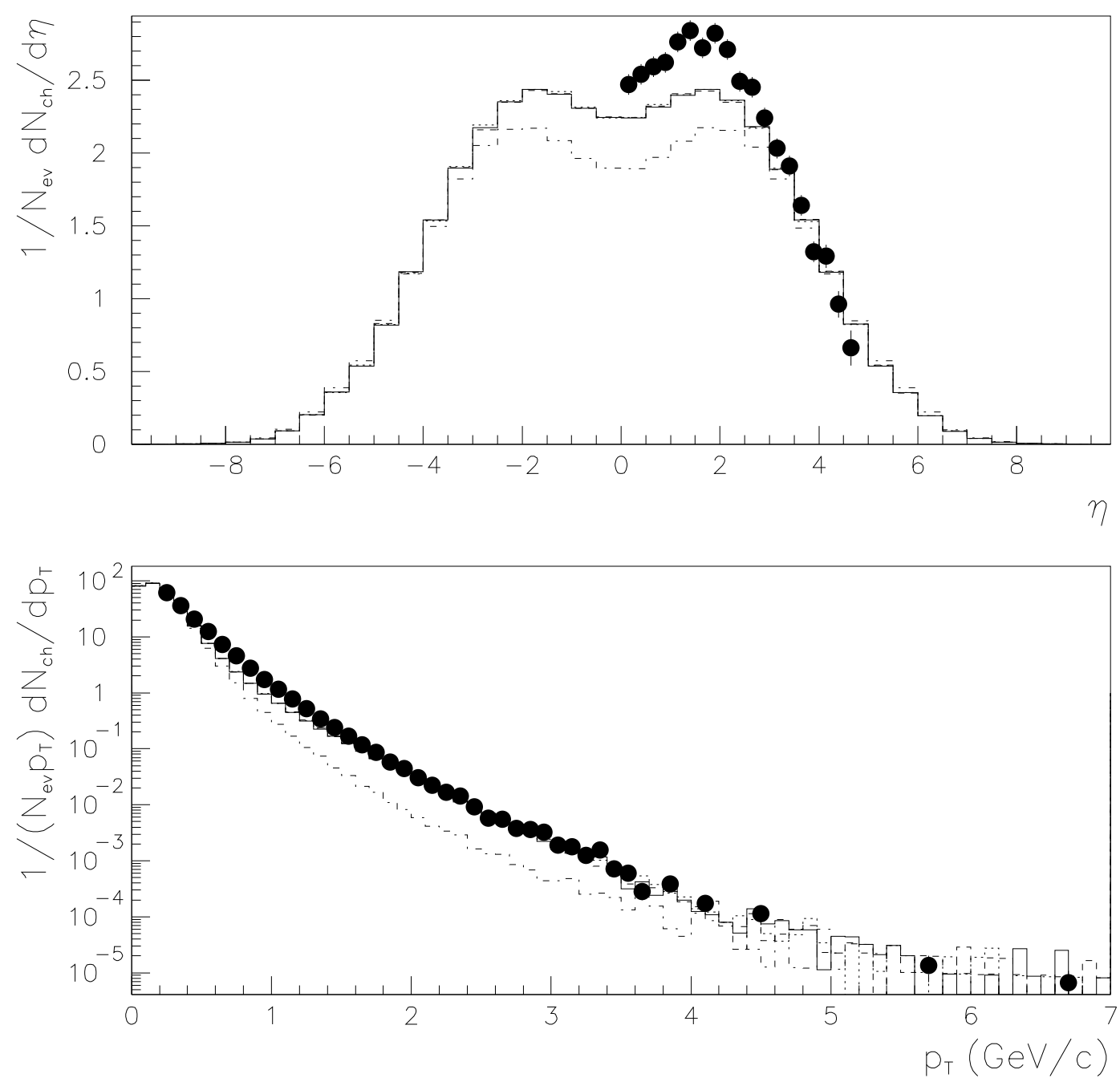

Figure 3: 

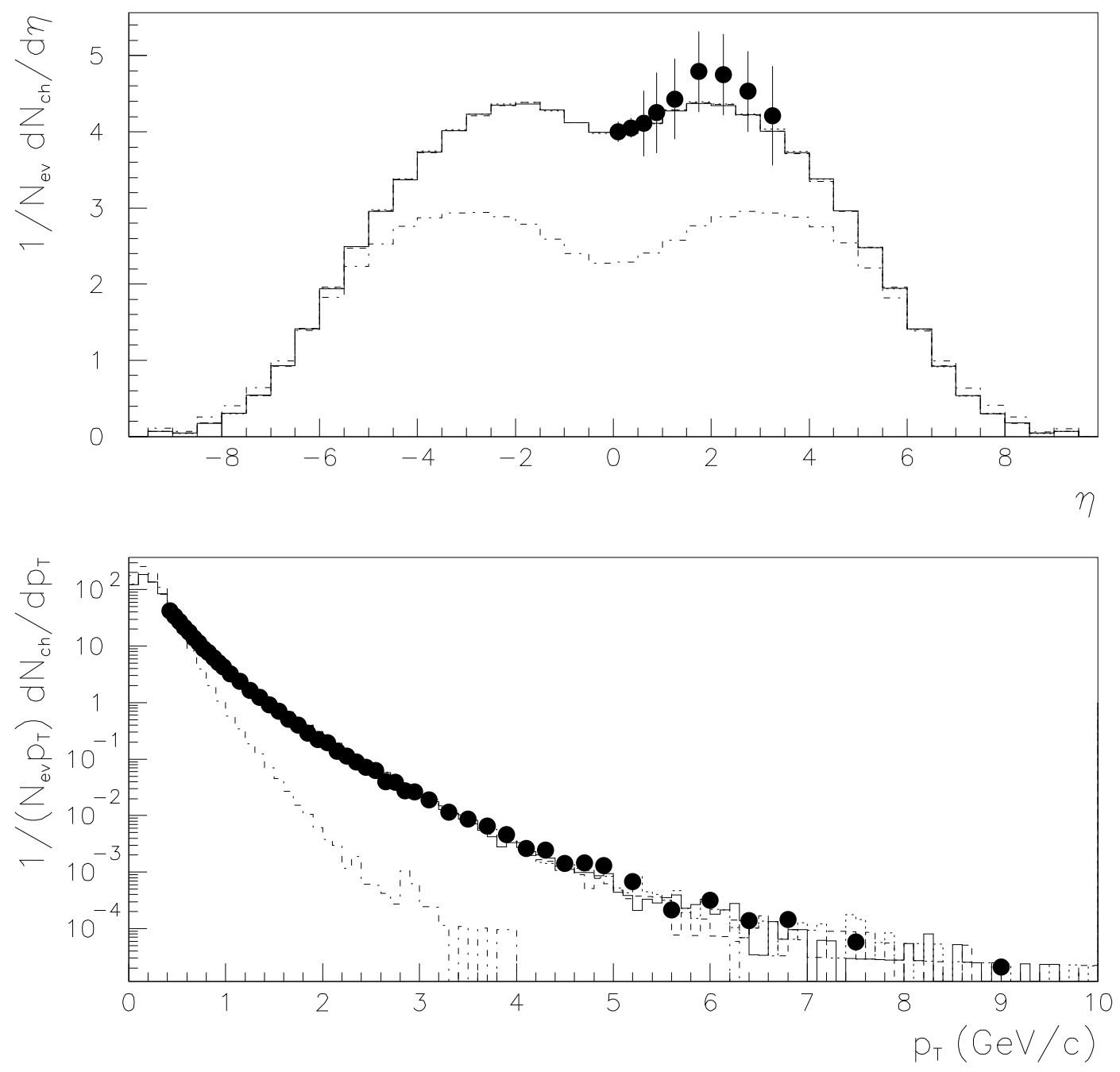

Figure 4: 

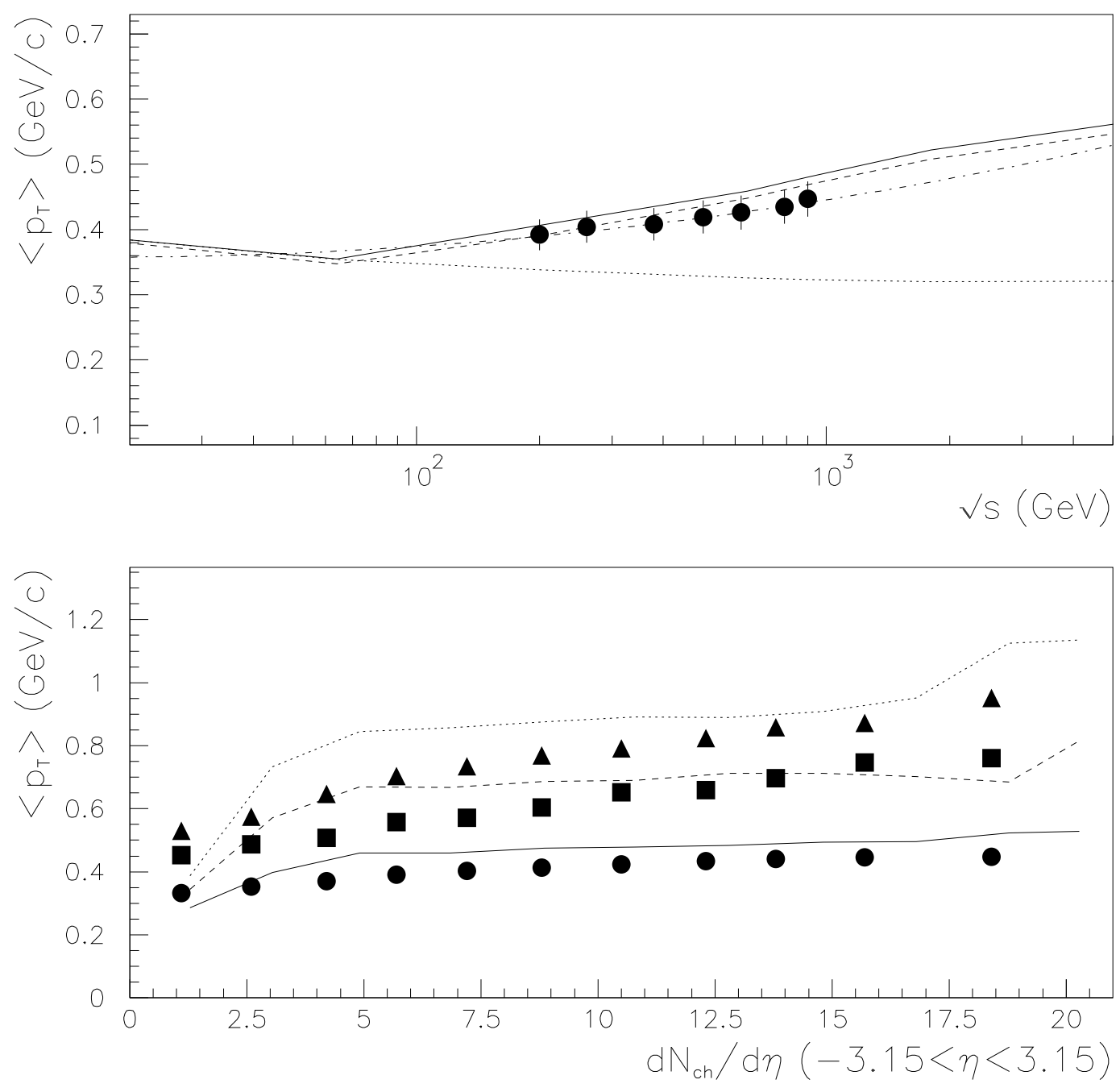

Figure 5: 


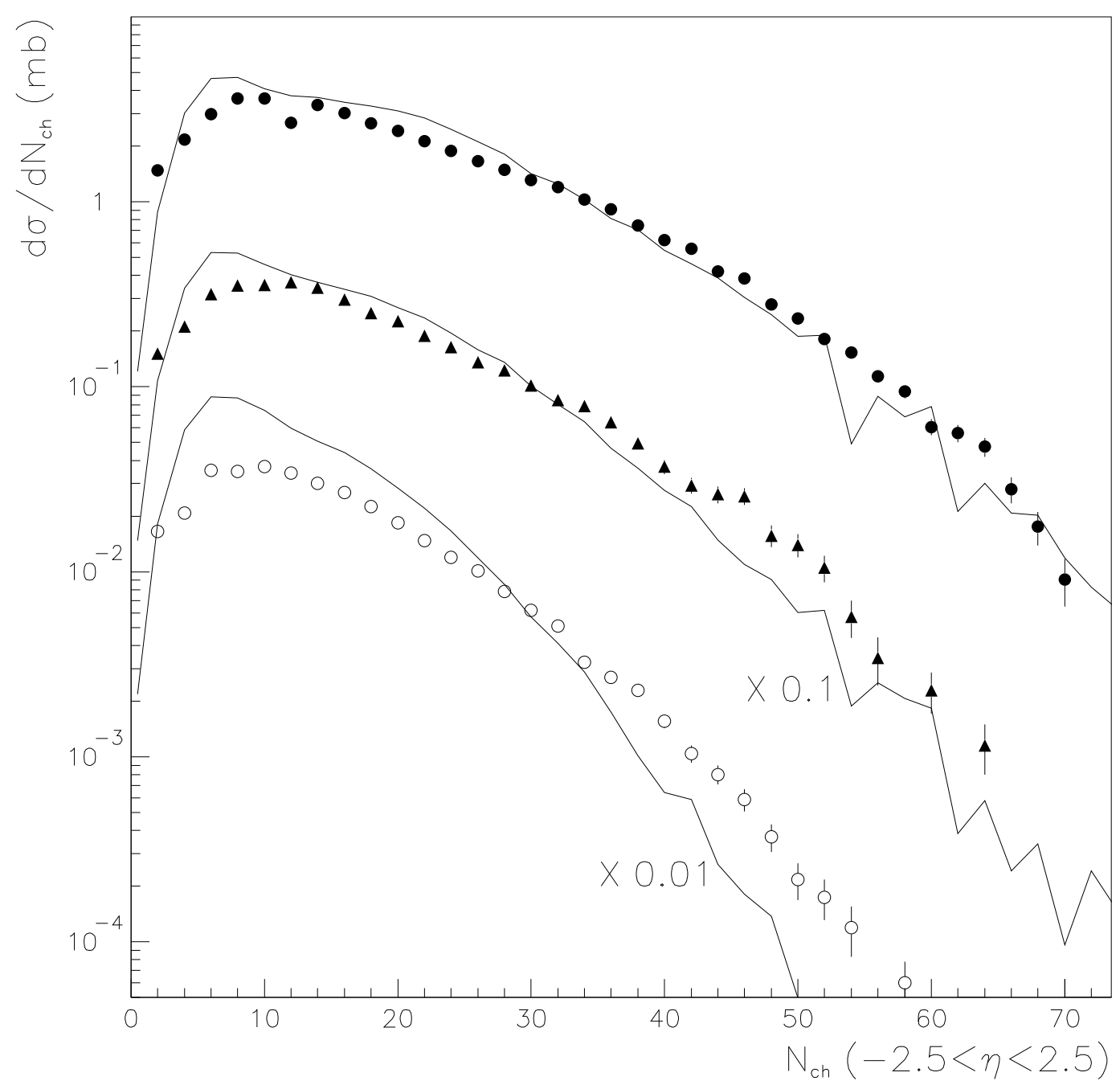

Figure 6: 

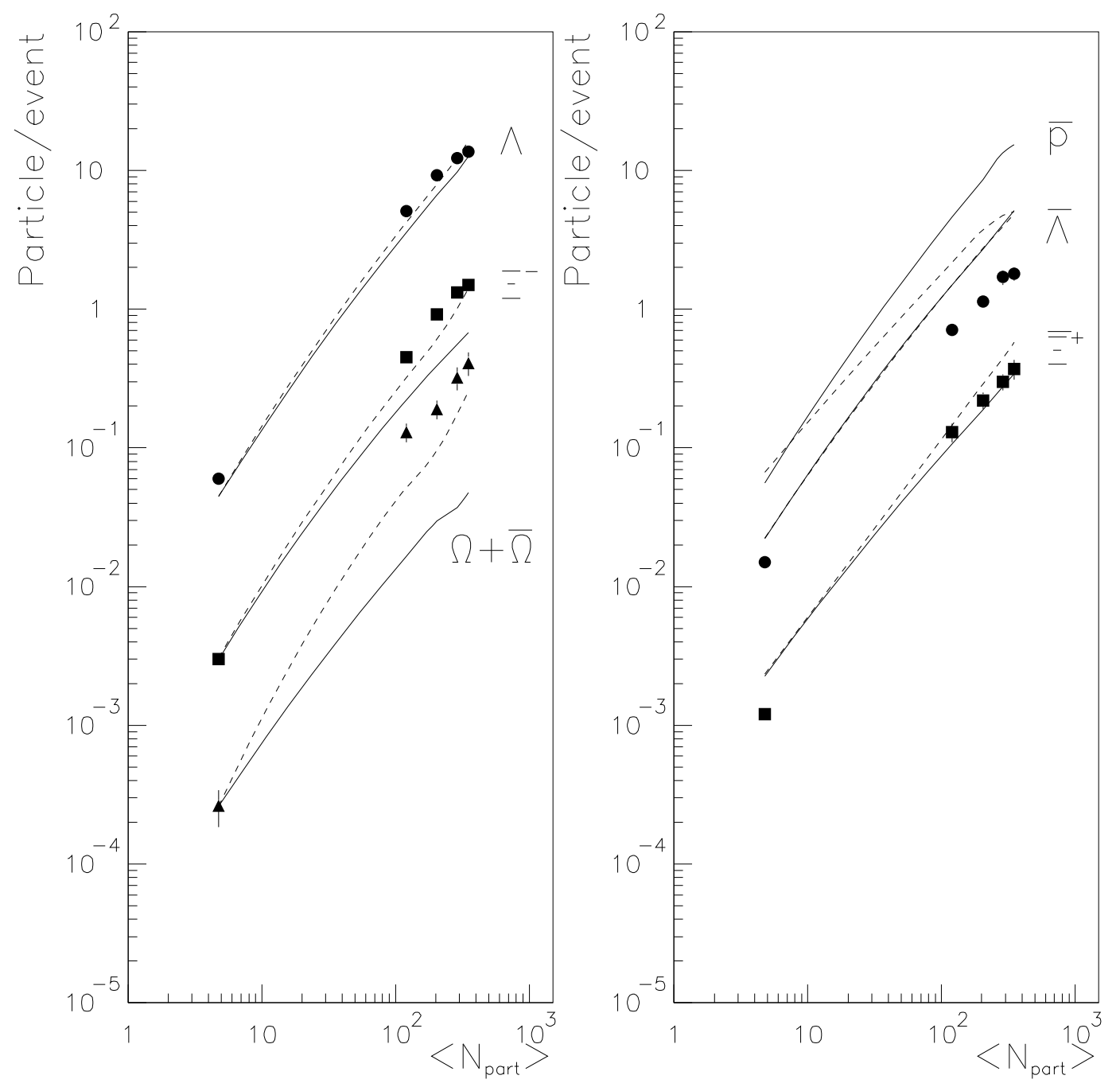

Figure 7: 

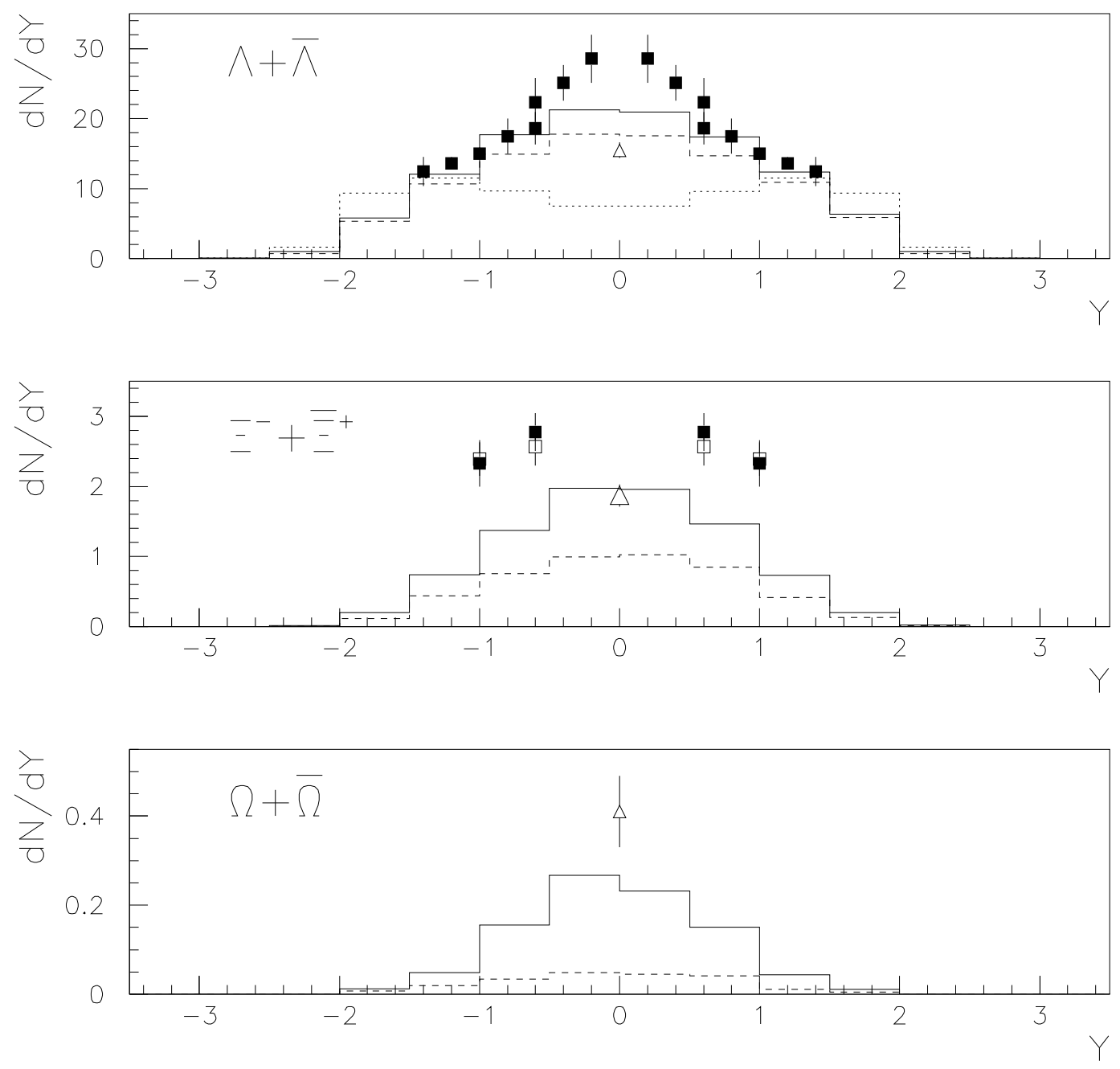

Figure 8: 


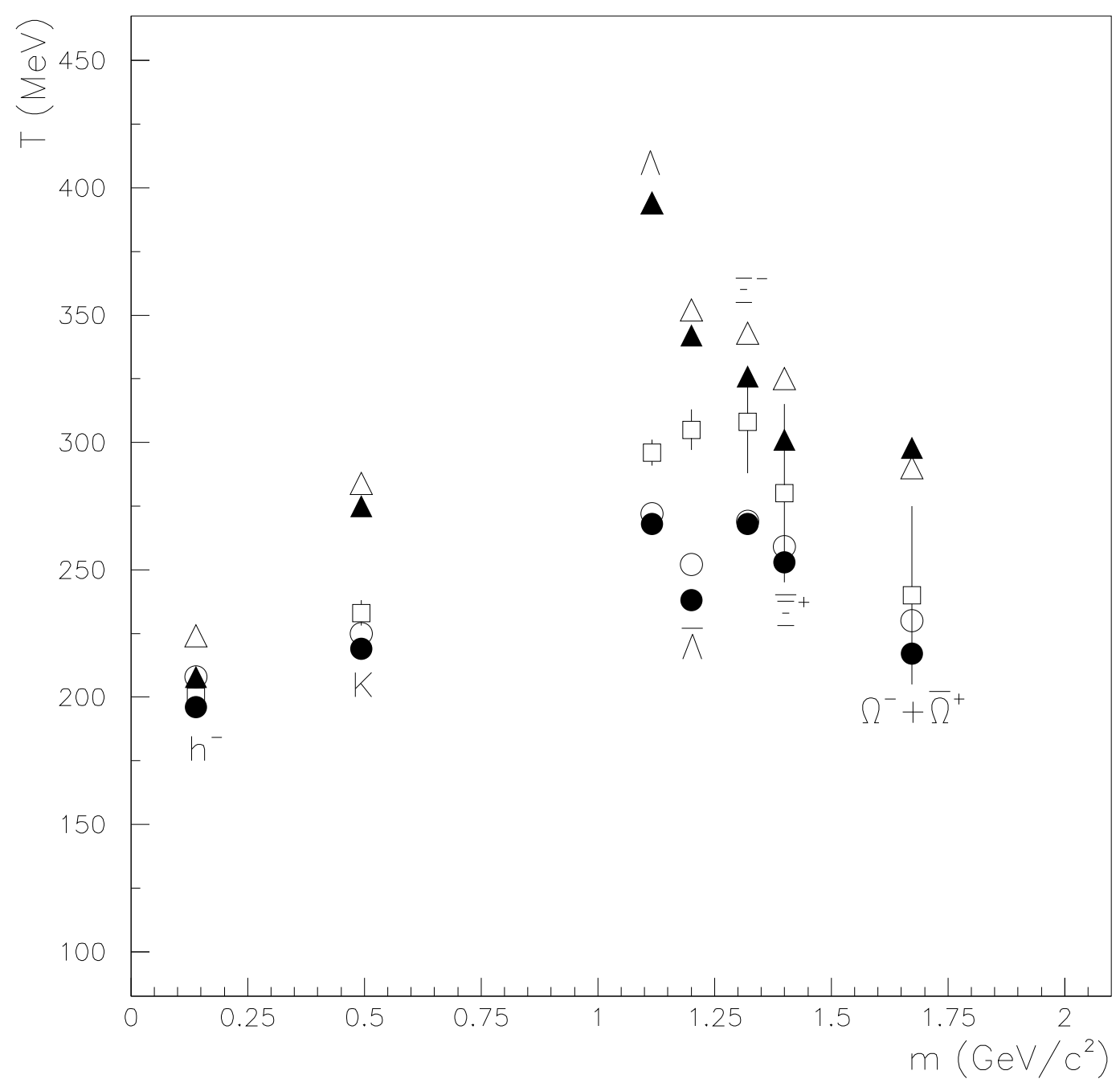

Figure 9: 

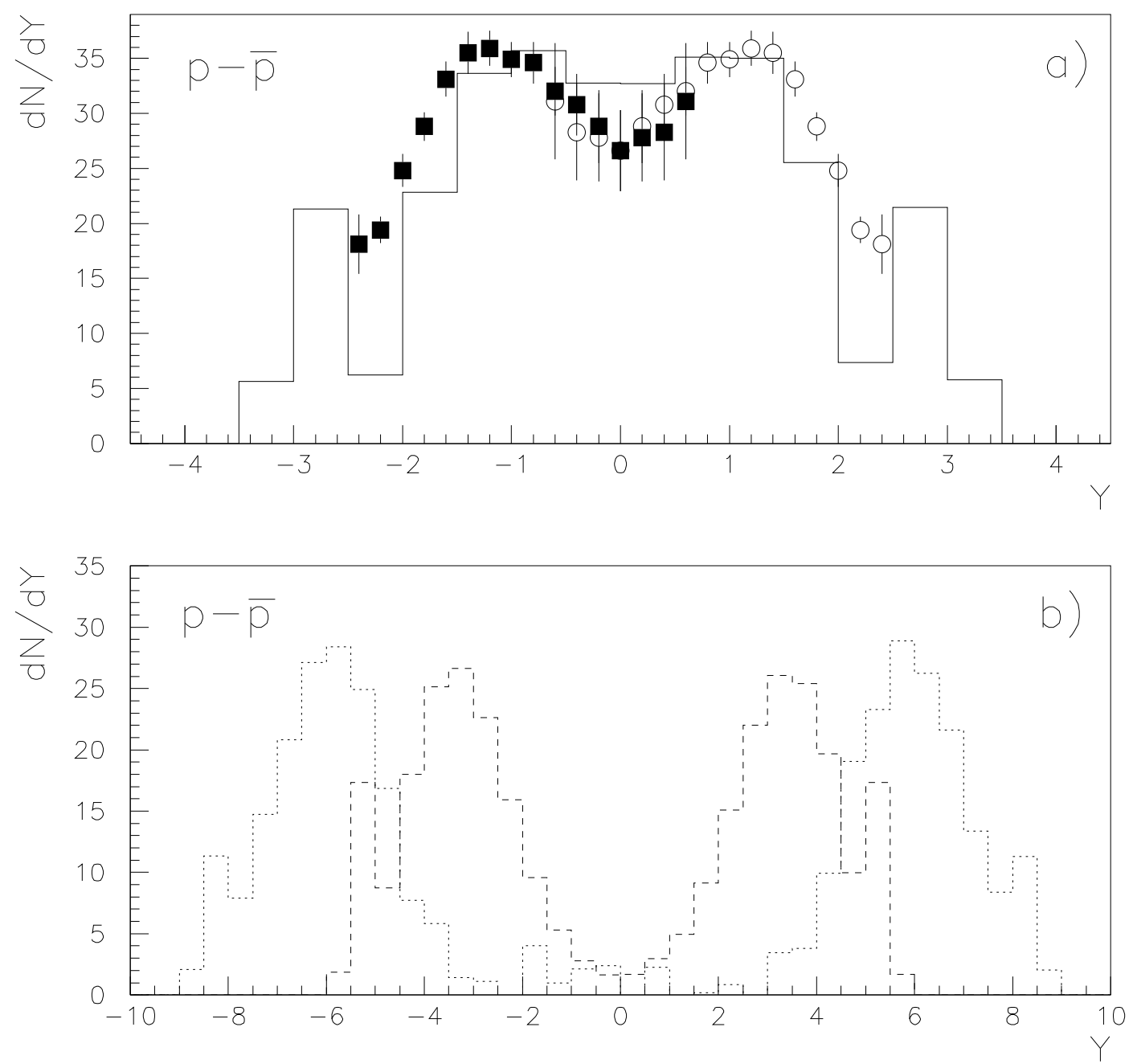

Figure 10: 


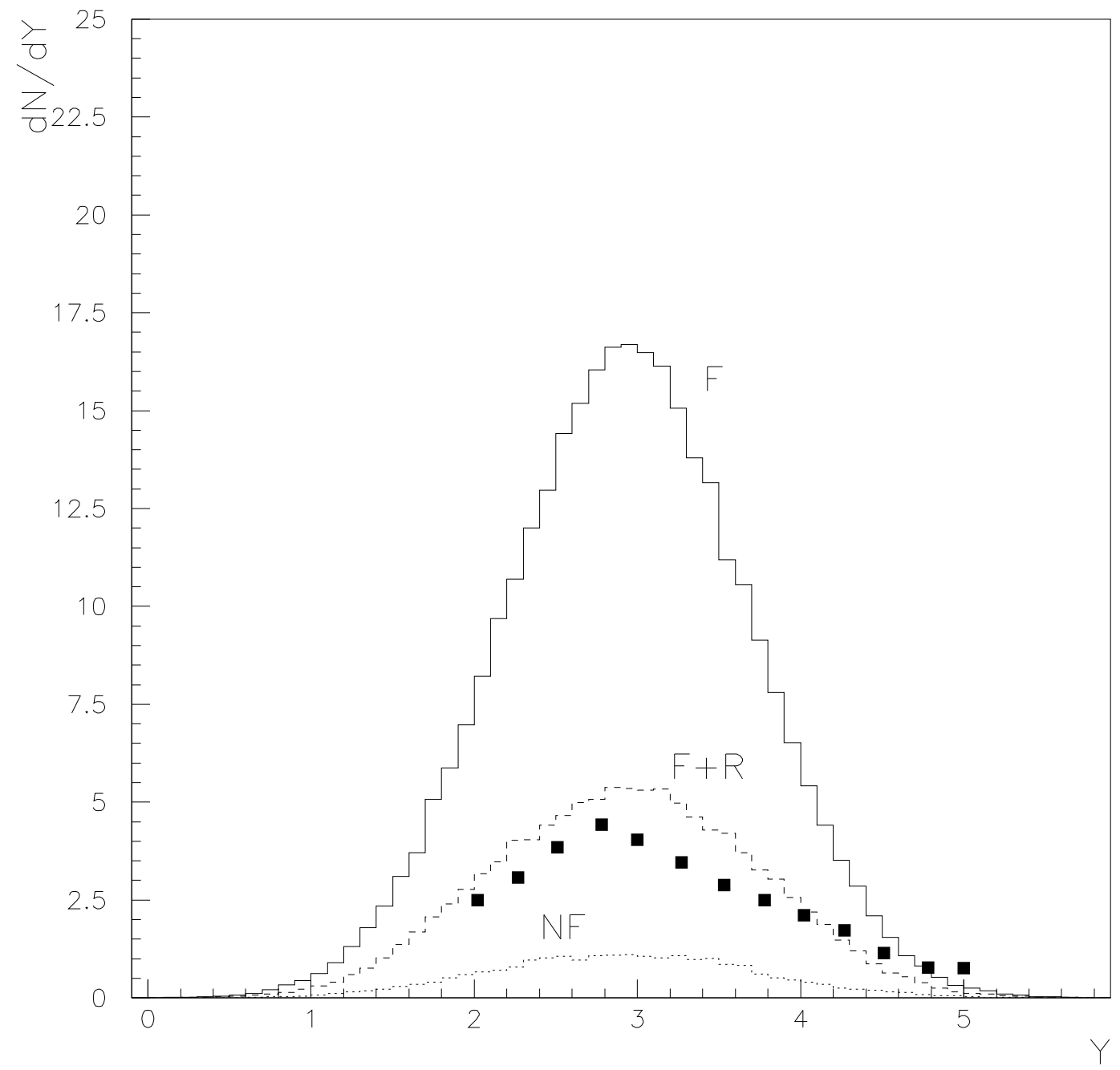

Figure 11: 


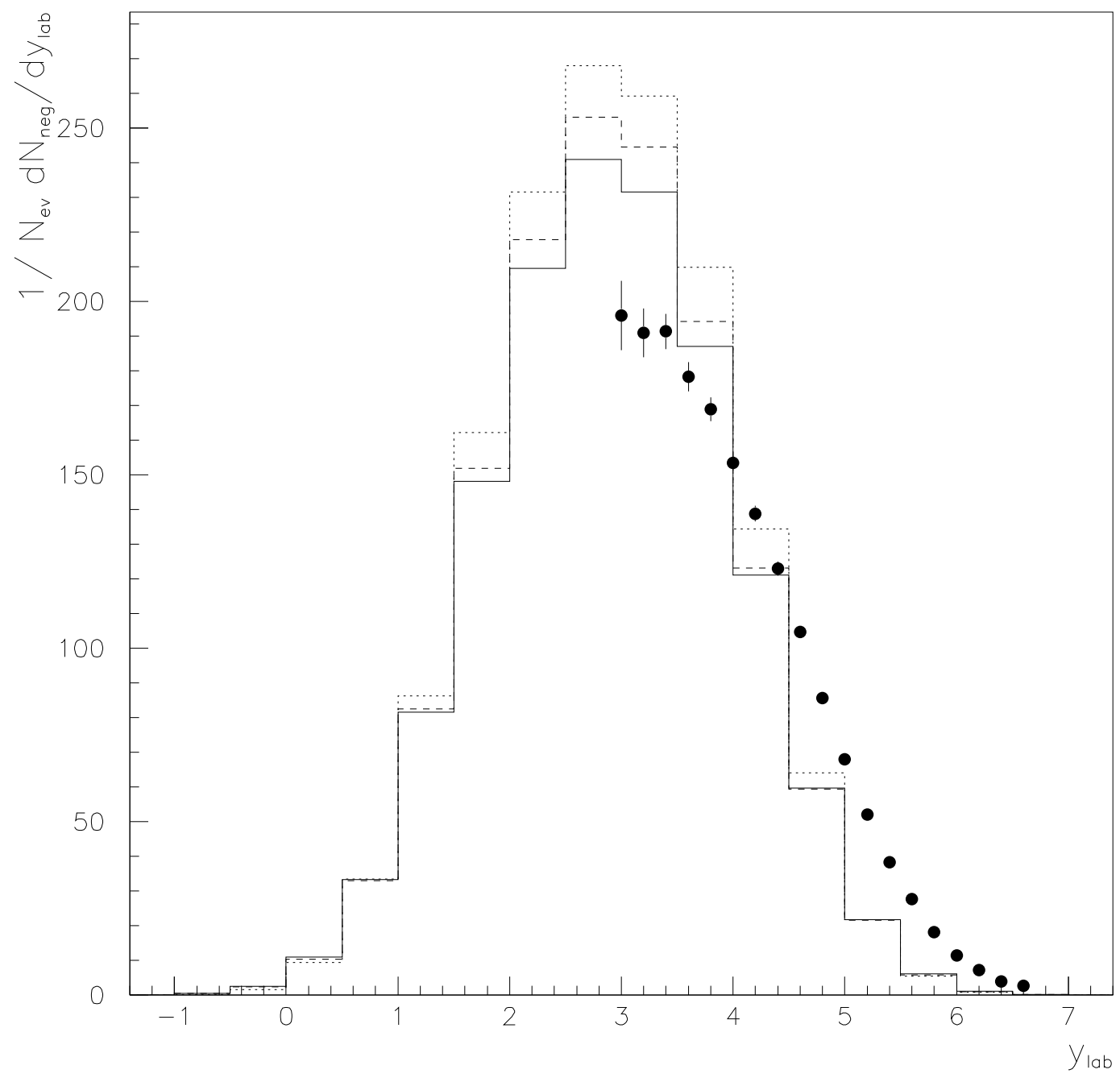

Figure 12: 

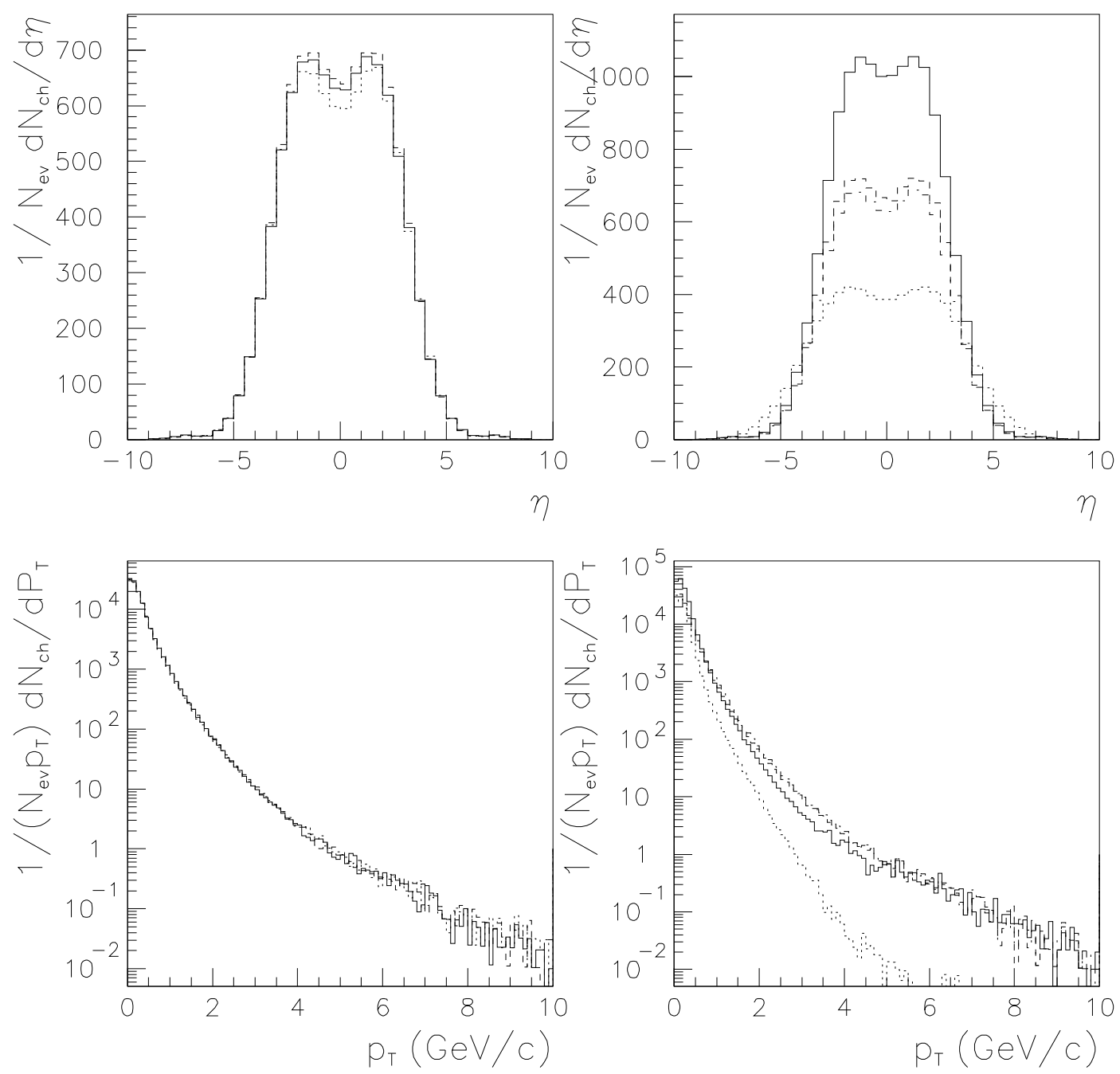

Figure 13: 

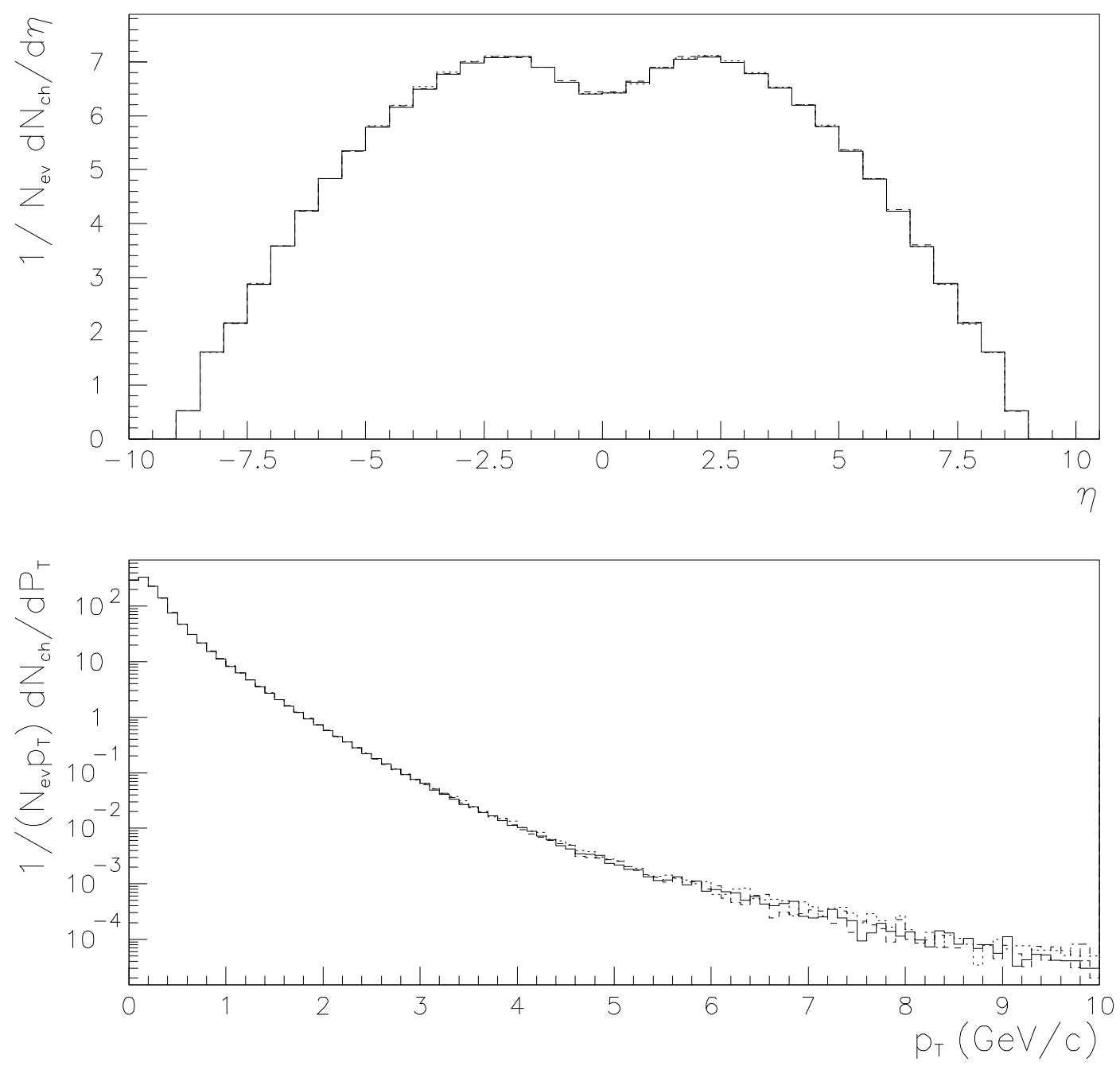

Figure 14: 

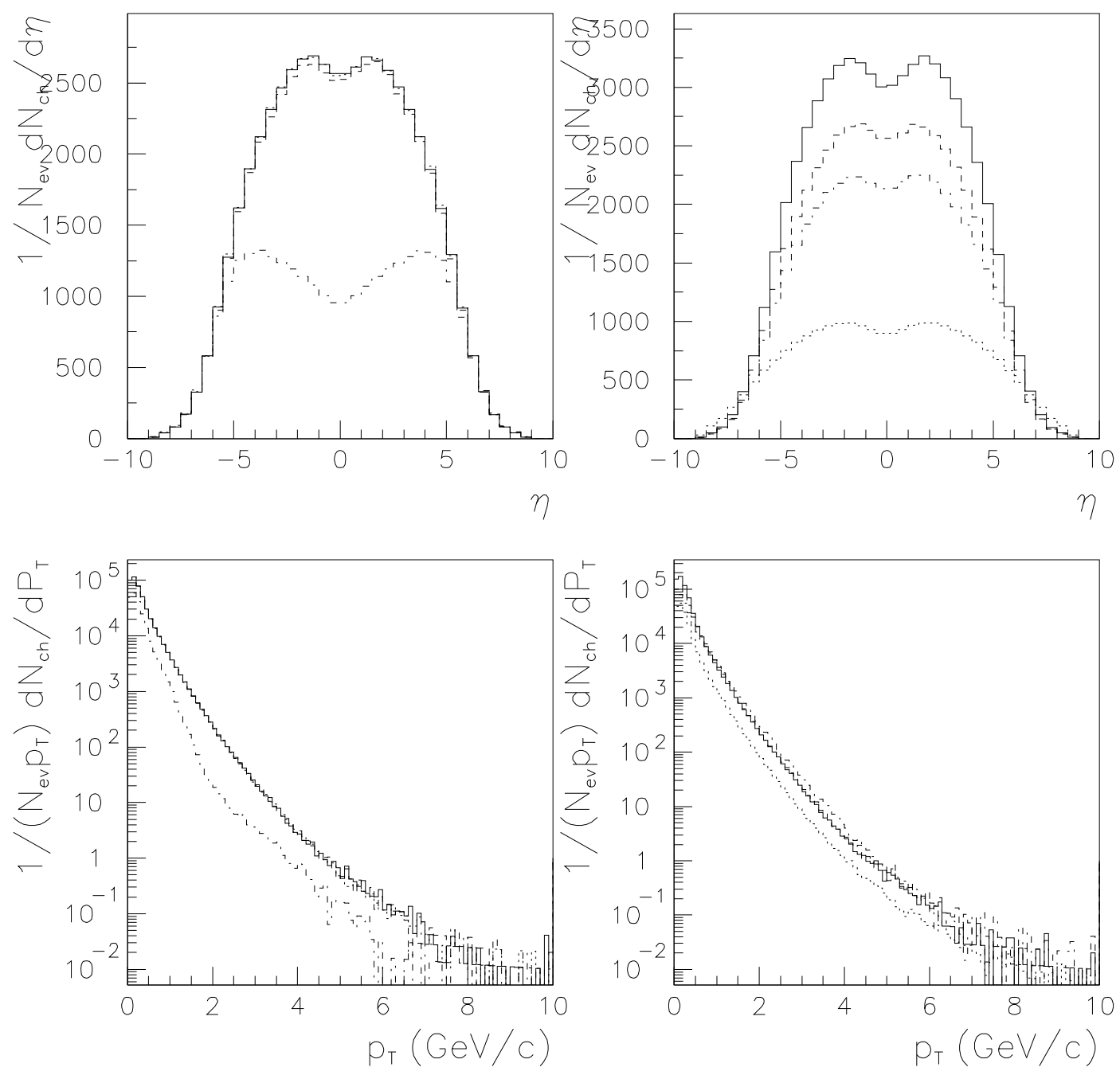

Figure 15: 
Tables:

\begin{tabular}{cccc}
\hline \hline & No fusion & Fusion & Experiment \\
\hline \hline charged & 7.89 & 7.81 & $7.69 \pm 0.06$ \\
\hline negatives & 2.95 & 2.90 & $2.85 \pm 0.03$ \\
\hline $\mathrm{p}$ & 1.18 & 1.19 & $1.34 \pm 0.15$ \\
\hline$\pi^{+}$ & 3.40 & 3.33 & $3.22 \pm 0.12$ \\
\hline$\pi^{-}$ & 2.69 & 2.63 & $2.62 \pm 0.06$ \\
\hline$\pi^{0}$ & 3.73 & 3.68 & $3.34 \pm 0.24$ \\
\hline$K^{+}$ & 0.31 & 0.32 & $0.28 \pm 0.06$ \\
\hline$K^{-}$ & 0.18 & 0.18 & $0.18 \pm 0.05$ \\
\hline$\Lambda$ & 0.223 & 0.231 & $0.096 \pm 0.010$ \\
\hline $\bar{\Lambda}$ & 0.029 & 0.033 & $0.0136 \pm 0.0040$ \\
\hline$\overline{\mathrm{p}}$ & 0.059 & 0.070 & $0.05 \pm 0.02$ \\
\hline \hline
\end{tabular}

Table 1: 


\begin{tabular}{cccc}
\hline \hline & No fusion & Fusion & Experiment \\
\hline \hline $\mathrm{p}$ & 1.20 & 1.21 & $1.20 \pm 0.12$ \\
\hline$\pi^{+}$ & 4.04 & 3.94 & $4.10 \pm 0.26$ \\
\hline$\pi^{-}$ & 3.32 & 3.23 & $3.34 \pm 0.20$ \\
\hline$\pi^{0}$ & 4.47 & 4.38 & $3.87 \pm 0.28$ \\
\hline$K^{+}$ & 0.38 & 0.38 & $0.33 \pm 0.02$ \\
\hline$K^{-}$ & 0.25 & 0.24 & $0.22 \pm 0.01$ \\
\hline$\Lambda$ & 0.245 & 0.251 & $0.13 \pm 0.01$ \\
\hline $\bar{\Lambda}$ & 0.045 & 0.049 & $0.020 \pm 0.005$ \\
\hline$\overline{\mathrm{p}}$ & 0.088 & 0.100 & $0.063 \pm 0.002$ \\
\hline \hline
\end{tabular}

Table 2:

\begin{tabular}{cccc}
\hline \hline & No fusion & Fusion & Experiment \\
\hline \hline $\mathrm{pS}$ & 5.01 & 4.86 & $5.10 \pm 0.20$ \\
\hline $\mathrm{pAr}$ & 5.31 & 5.12 & $5.39 \pm 0.17$ \\
\hline $\mathrm{pAg}$ & 6.57 & 6.28 & $6.2 \pm 0.2$ \\
\hline $\mathrm{pXe}$ & 6.89 & 6.56 & $6.84 \pm 0.13$ \\
\hline $\mathrm{pAu}$ & 7.54 & 7.16 & $7.0 \pm 0.4$ \\
\hline \hline
\end{tabular}

Table 3: 


\begin{tabular}{ccccc}
\hline \hline & NF & F & FR & Experiment \\
\hline \hline negatives & 108.2 & 101.3 & 100.7 & $98 \pm 3$ \\
\hline$K^{+}$ & 9.7 & 10.4 & 10.8 & $12.5 \pm 0.4$ \\
\hline$K^{-}$ & 7.1 & 7.2 & 7.4 & $6.9 \pm 0.4$ \\
\hline$\Lambda$ & 5.0 & 5.9 & 6.0 & $9.4 \pm 1.0$ \\
\hline $\bar{\Lambda}$ & 0.4 & 1.1 & 1.2 & $2.2 \pm 0.4$ \\
\hline$\overline{\mathrm{p}}$ & 0.82 & 3.23 & 2.80 & \\
\hline$\Xi^{-}$ & 0.024 & 0.186 & 0.205 & \\
\hline $\bar{\Xi}^{+}$ & 0.028 & 0.097 & 0.102 & \\
\hline$\Omega^{-}$ & 0.001 & 0.007 & 0.010 & \\
\hline $\bar{\Omega}^{+}$ & 0.001 & 0.005 & 0.007 & \\
\hline \hline
\end{tabular}

Table 4:

\begin{tabular}{ccccc}
\hline \hline & NF & F & FR & Experiment \\
\hline \hline $\bar{\Lambda} / \Lambda$ & 0.14 & 0.41 & 0.34 & $0.128 \pm 0.012$ \\
\hline $\bar{\Xi}^{+} / \Xi^{-}$ & 1.12 & 0.52 & 0.45 & $0.266 \pm 0.028$ \\
\hline $\bar{\Omega}^{+} / \Omega^{-}$ & 0.75 & 0.88 & 0.59 & $0.46 \pm 0.15$ \\
\hline$\Xi^{-} / \Lambda$ & 0.01 & 0.06 & 0.08 & $0.093 \pm 0.007$ \\
\hline$\Xi^{+} / \bar{\Lambda}$ & 0.08 & 0.07 & 0.10 & $0.195 \pm 0.023$ \\
\hline$\Omega / \Xi$ & 0.05 & 0.05 & 0.11 & $0.195 \pm 0.028$ \\
\hline \hline
\end{tabular}

Table 5: 


\begin{tabular}{cccccccc}
\hline \hline & RHIC(F) & RHIC(FR) & QCM & Rafelski & B-M & LHC(F) & LHC(FR $)$ \\
\hline \hline $\bar{\Lambda} / \Lambda$ & 1.01 & 0.90 & 0.69 & $0.49 \pm 0.15$ & 0.91 & 1.00 & 0.98 \\
\hline $\bar{\Xi}^{+} / \Xi^{-}$ & 0.96 & 0.97 & 0.83 & $0.68 \pm 0.15$ & 1.0 & 0.98 & 0.95 \\
\hline $\bar{\Omega}^{+} / \Omega^{-}$ & 1.00 & 1.25 & 1.0 & 1.0 & 1.0 & 0.76 & 1.03 \\
\hline$\Xi^{-} / \Lambda$ & 0.10 & 0.15 & - & $0.18 \pm 0.02$ & 0.13 & 0.09 & 0.25 \\
\hline $\bar{\Xi}^{+} / \bar{\Lambda}$ & 0.10 & 0.16 & - & $0.25 \pm 0.03$ & 0.14 & 0.09 & 0.24 \\
\hline$\Omega / \Xi$ & 0.07 & 0.26 & - & $0.14 \pm 0.03$ & 0.20 & 0.05 & 0.40 \\
\hline $\bar{\Lambda} / \bar{p}$ & 0.40 & 0.71 & - & $2.4 \pm 0.3$ & 0.52 & 0.35 & 0.82 \\
\hline $\bar{p} / p$ & 0.93 & 0.90 & 0.58 & $0.34_{-0.12}^{+0.37}$ & 0.90 & 1.00 & 1.04 \\
\hline \hline
\end{tabular}

Table 6: 